TRANSACTIONS OF THE

AMERICAN MATHEMATICAL SOCIETY

Volume 355, Number 8, Pages 3143-3174

S 0002-9947(03)03285- 9

Article electronically published on April 11, 2003

\title{
A GENERALIZATION OF TIGHT CLOSURE AND MULTIPLIER IDEALS
}

\author{
NOBUO HARA AND KEN-ICHI YOSHIDA
}

\begin{abstract}
We introduce a new variant of tight closure associated to any fixed ideal $\mathfrak{a}$, which we call $\mathfrak{a}$-tight closure, and study various properties thereof. In our theory, the annihilator ideal $\tau(\mathfrak{a})$ of all $\mathfrak{a}$-tight closure relations, which is a generalization of the test ideal in the usual tight closure theory, plays a particularly important role. We prove the correspondence of the ideal $\tau(\mathfrak{a})$ and the multiplier ideal associated to $\mathfrak{a}$ (or, the adjoint of $\mathfrak{a}$ in Lipman's sense) in normal $\mathbb{Q}$-Gorenstein rings reduced from characteristic zero to characteristic $p \gg 0$. Also, in fixed prime characteristic, we establish some properties of $\tau(\mathfrak{a})$ similar to those of multiplier ideals (e.g., a Briançon-Skoda-type theorem, subadditivity, etc.) with considerably simple proofs, and study the relationship between the ideal $\tau(\mathfrak{a})$ and the F-rationality of Rees algebras.
\end{abstract}

\section{INTRODUCTION}

The notion of tight closure, introduced by Hochster and Huneke [HH1] more than a decade ago, has emerged as a powerful new tool in commutative algebra. Tight closure gives remarkably simple characteristic $p$ proofs of several results that were not thought to be particularly related, e.g., that rings of invariants of linearly reductive groups acting on regular rings are Cohen-Macaulay, that the integral closure of the $n$th power of an $n$-generator ideal of a regular ring is contained in the ideal (the Briançon-Skoda theorem), and so on. Also, the notions of F-regular and F-rational rings are defined via tight closure, and they turned out to correspond to log terminal and rational singularities, respectively ([Ha1, HW], [MS], Sm1]). This result is generalized to the correspondence of test ideals and multiplier ideals ( and birational algebraic geometry, respectively.

The test ideal of a ring $R$ of characteristic $p$, denoted by $\tau(R)$, is the annihilator ideal of all tight closure relations of $R$. On the other hand, the notion of multiplier ideals has several variants. Originally, a multiplier ideal was defined analytically for a given plurisubharmonic function on a complex analytic manifold [N]. This is reformulated in the algebro-geometric setting (in characteristic zero) in terms of resolution of singularities and discrepancy divisors ([Ei], [La] $)$. Actually, two types of multiplier ideals are defined in this setting, that is, the multiplier ideal $\mathcal{J}(D)$ associated to a $\mathbb{Q}$-divisor $D$ and the multiplier ideal $\mathcal{J}(\mathfrak{a})$ associated to an ideal $\mathfrak{a}$.

Received by the editors August 20, 2002 and, in revised form, December 19, 2002.

2000 Mathematics Subject Classification. Primary 13A35, 14B05.

Both authors are partially supported by a Grant-in-Aid for Scientific Research, Japan.

(C)2003 American Mathematical Society 
The latter is also defined by Lipman [Li] in a more algebraic context and is called the "adjoint ideal".

Precisely speaking, the multiplier ideal that is proved to correspond to the test ideal $\tau(R)$ is the one associated to the trivial divisor $D=0$ or the unit ideal $\mathfrak{a}=R$, which defines the non-log-terminal locus of $\operatorname{Spec} R$. In most applications, however, the usefulness of multiplier ideals is obtained by considering multiplier ideals associated to various divisors or ideals; see, e.g., [Ei], [La, Li]. Thus we are tempted to define a sort of tight closure operation and a "test ideal" associated to any given $\mathbb{Q}$-divisor or ideal.

In this paper, we introduce a generalization of tight closure, which we call $\mathfrak{a}$-tight closure, associated to an ideal $\mathfrak{a}$, study various properties (including the relationship with multiplier ideals), and give some applications of a-tight closure. (In [T], the reader can find an attempt to generalize tight closure in the other direction, that is, $\Delta$-tight closure associated to a $\mathbb{Q}$-divisor $\Delta$; see also $[\mathrm{HW}$.) Actually, given an ideal $\mathfrak{a}$ of a Noetherian ring $R$ of characteristic $p>0$, we define the $\mathfrak{a}$-tight closure $I^{* \mathfrak{a}}$ of an ideal $I \subseteq R$ to be the ideal consisting of all elements $z \in R$ for which there exists an element $c \in R$ not in any minimal prime ideal such that

$$
c z^{p^{e}} \mathfrak{a}^{p^{e}} \subseteq I^{\left[p^{e}\right]}
$$

for all $e \gg 0$, where $I^{\left[p^{e}\right]}$ is the ideal generated by the $p^{e}$ th powers of elements of $I$; see Definition 1.1 and also Definition 6.1 for further generalization to "rational coefficients". We then define the ideal $\tau(\mathfrak{a})$ of $R$ to be the unique largest ideal such that $\tau(\mathfrak{a}) I^{* \mathfrak{a}} \subseteq I$ for all ideals $I \subseteq R$. So, in the case where $\mathfrak{a}=R$ is the unit ideal, the $\mathfrak{a}$-tight closure $I^{* \mathfrak{a}}=I^{* R}$ is equal to the tight closure $I^{*}$ in the usual sense, and the ideal $\tau(\mathfrak{a})=\tau(R)$ is nothing but the test ideal [HH1].

There are many similarities between the usual tight closure and $\mathfrak{a}$-tight closure. For example, the existence of a-test elements (Definition 1.6) is proved quite similarly as in the case for the usual test elements. But there does exist a difference as well: We require a "closure" operation to satisfy the property that, once the operation is performed, the obtained closure does not change if one performs the operation twice or more, and tight closure satisfies this property, namely, $\left(I^{*}\right)^{*}=I^{*}$. However, it happens that $\left(I^{* \mathfrak{a}}\right)^{* \mathfrak{a}}$ is strictly larger than $I^{* \mathfrak{a}}$, and so, a-tight closure is not in fact a closure operation. Similarly, unlike the usual test ideal $\tau(R)$, the ideal $\tau(\mathfrak{a})$ is no longer equal to the one generated by $\mathfrak{a}$-test elements if $\mathfrak{a} \subsetneq R$.

In spite of the apparent disadvantage mentioned above, we find many more advantages in the circle of ideas involving $\mathfrak{a}$-tight closure. The significance of the ideal $\tau(\mathfrak{a})$ is witnessed by the following theorem, which ensures the expected correspondence of $\tau(\mathfrak{a})$ and the multiplier ideal $\mathcal{J}(\mathfrak{a})$; see also Theorem 6.7.

Theorem 3.4. Let $R$ be a normal $\mathbb{Q}$-Gorenstein local ring essentially of finite type over a field, and let $\mathfrak{a}$ be a nonzero ideal. Assume that $\mathfrak{a} \subseteq R$ is reduced from characteristic zero to characteristic $p \gg 0$, together with a log resolution of singularities $f: X \rightarrow Y=\operatorname{Spec} R$ such that $\mathfrak{a} \mathcal{O}_{X}=\mathcal{O}_{X}(-Z)$ is invertible. Then

$$
\tau(\mathfrak{a})=H^{0}\left(X, \mathcal{O}_{X}\left(\left\lceil K_{X}-f^{*} K_{Y}\right\rceil-Z\right)\right) .
$$

Note that, by definition, the multiplier ideal $\mathcal{J}(\mathfrak{a})$ in characteristic zero takes just the same form as the right-hand side of the above equality. So one can think of the right-hand side as a reduction modulo $p$ of the multiplier ideal in characteristic zero. This theorem generalizes the main results of [Ha2] and [Sm2], and is proved essentially in a similar way as in those papers, with a little more effort. 
The usefulness of multiplier ideals is tied up with vanishing theorems in characteristic zero such as the Nadel vanishing theorem [N], which fail in characteristic $p>0$. For example, Lipman used these tools to establish an improved version of the Briançon-Skoda theorem, which asserts that, for any ideal $\mathfrak{a}$ of a regular local ring generated by $r$ elements, one has $\mathcal{J}\left(\mathfrak{a}^{n+r-1}\right) \subseteq \mathfrak{a}^{n}$ for all $n \geq 0$ [Li]; see also [La]. We will prove in Theorem 2.1 that the corresponding statement $\tau\left(\mathfrak{a}^{n+r-1}\right) \subseteq \mathfrak{a}^{n}$ holds true in characteristic $p>0$. Taking into account the correspondence in Theorem 3.4, we see that this gives another proof of Lipman's result. An advantage of our prime characteristic proof here is that it is quite elementary and simple (like the original tight closure proof of Briançon-Skoda [HH1]) and does not depend on desingularization or vanishing theorems.

We shall take a look at the organization of this paper, which we hope gives further confirmation of the usefulness of $\mathfrak{a}$-tight closure.

After discussing basic properties of $\mathfrak{a}$-tight closure and the ideal $\tau(\mathfrak{a})$ in Section 1, we give three fundamental applications of a-tight closure in Section 2. The first one is the modified Briançon-Skoda theorem via a-tight closure mentioned above. Second, we study the relationship between $\mathfrak{a}$-tight closure and tight integral closure defined by Hochster [Ho2], and rephrase the F-rationality criterion of Rees algebras obtained in [HWY1] in terms of a-tight closure; see Theorem 2.7 and Corollary 2.9. This enables us to give an interesting characterization of regular local rings as the third application. Namely, we prove in Theorem 2.15 that the regularity of a $d$-dimensional local ring $(R, \mathfrak{m})$ is characterized by the property that $\tau\left(\mathfrak{m}^{d-1}\right)=$ $R$. This is considered an analog of the fact that the weak F-regularity of $R$ is characterized by the property that $\tau(R)=R$.

Section 3 is devoted to proving the equality $\tau(\mathfrak{a})=\mathcal{J}(\mathfrak{a})$ in Theorem 3.4, which holds true in the situation reduced from characteristic zero to characteristic $p \gg 0$. We note that the containment $\tau(\mathfrak{a}) \subseteq \mathcal{J}(\mathfrak{a})$ essentially holds true in any fixed characteristic $p>0$; cf. Proposition 3.8.

In Section 4, we establish properties of ideals $\tau(\mathfrak{a})$ in fixed characteristic $p>0$ similar to those of multiplier ideals $\mathcal{J}(\mathfrak{a})$ in characteristic zero, namely, the restriction theorem (Theorem 4.1), the subadditivity (Theorem 4.5), and a description of ideals $\tau(\mathfrak{a})$ in the toric case (Theorem 4.8); see DEL, How, La for the results proved for multiplier ideals. Again in light of Theorem 3.4, we can also say that the results in this section give new prime characteristic proofs of the geometric statements for multiplier ideals in characteristic zero, although we work with the Frobenius map in fixed characteristic $p>0$.

In Section 5, we explore the behavior of the ideal $\tau(I)$ for an $\mathfrak{m}$-primary ideal $I$ of a Gorenstein local domain $(R, \mathfrak{m})$ of characteristic $p>0$, from the viewpoint of the Rees algebra $\mathbf{R}(I)=R[I t]$ via Theorem 2.7. As a main result of this section, we prove in Theorem 5.1 that if $\mathbf{R}(I)$ is F-rational, then its graded canonical module is described as $\omega_{\mathbf{R}(I)}=\bigoplus_{n \geq 1} \tau\left(I^{n}\right) t^{n}$. In particular, the equality $\tau(I)=\mathcal{J}(I)$ holds if $\mathbf{R}(I)$ is F-rational, and the converse is also true in the case of a twodimensional rational double point. (It should be noted that the ideals $\tau(I)$ and $\mathcal{J}(I)$ may disagree in fixed positive characteristic.) Comparing Theorem 5.1 with Hyry's results ([Hy1], Hy2]), we can also deduce various results for $\tau(I)$.

In Section 6, we extend the notions of $\mathfrak{a}$-tight closure and the ideal $\tau(\mathfrak{a})$ to those with "rational coefficients", and generalize some results discussed in the previous sections to the case of rational coefficients. Although we have no explicit applica- 
tions of this generalization at the moment, we include this section for future reference, because recent applications of multiplier ideals involve rational coefficients successfully ([ELS], [La]).

Before going ahead, we review part of the notation and basic notions of the tight closure theory. We keep it minimal to avoid overlap with Section 1. The reader is referred to Hochster and Huneke [HH1]-[HH3] and Huneke [Hu] for the full development of the theory.

Notation and basic notions. Throughout this paper all rings are Noetherian commutative rings with unity. For a ring $R$, we denote by $R^{\circ}$ the set of elements of $R$ that are not in any minimal prime ideal. We will often work over a field of characteristic $p>0$. In this case we always use the letter $q$ for a power $p^{e}$ of $p$.

Let $R$ be a Noetherian ring of characteristic $p>0$. For an ideal $I$ of $R$ and a power $q$ of $p$, we denote by $I^{[q]}$ the ideal generated by the $q$ th powers of elements of $I$. The tight closure $I^{*}$ of $I$ is defined to be the ideal consisting of all elements $z \in R$ for which there exists an element $c \in R^{\circ}$ such that $c z^{q} \in I^{[q]}$ for all large $q=p^{e}$. (Tight closure is also defined for a submodule of a module; cf. Section 1.)

We say that $R$ is weakly F-regular if every ideal $I$ of $R$ is tightly closed, that is, $I^{*}=I$. A local ring $(R, \mathfrak{m})$ is said to be F-rational if every ideal generated by a system of parameters of $R$ is tightly closed. In general, we say that $R$ is $F$-rational (resp. F-regular) if all of its local rings are F-rational (resp. weakly F-regular).

Let $F: R \rightarrow R$ be the Frobenius map, that is, the ring homomorphism sending $z \in R$ to $z^{p} \in R$. The ring $R$ viewed as an $R$-module via the $e$-times iterated Frobenius map $F^{e}: R \rightarrow R$ is denoted by ${ }^{e} R$. We say that $R$ is $F$-finite if ${ }^{1} R$ is a finitely generated $R$-module. If $R$ is reduced, then $F^{e}: R \rightarrow{ }^{e} R$ is identified with the natural inclusion map $R \hookrightarrow R^{1 / q}$. An F-finite reduced ring $R$ is said to be strongly F-regular if for every element $c \in R^{\circ}$, there exists a power $q=p^{e}$ such that the inclusion map $c^{1 / q} R \hookrightarrow R^{1 / q}$ splits as an $R$-module homomorphism.

\section{Definition and basic properties of a-tight Closure}

Let $R$ be a Noetherian ring of characteristic $p>0$ and let $M$ be an $R$-module. For each $e \in \mathbb{N}$, we define $\mathbb{F}^{e}(M)=\mathbb{F}_{R}^{e}(M):={ }^{e} R \otimes_{R} M$ and regard it as an $R$ module by the action of $R={ }^{e} R$ from the left. Then we have the induced $e$-times iterated Frobenius map $F^{e}: M \rightarrow \mathbb{F}^{e}(M)$. The image of $z \in M$ via this map is denoted by $z^{q}:=F^{e}(z) \in \mathbb{F}^{e}(M)$. For an $R$-submodule $N$ of $M$, we denote by $N_{M}^{[q]}$ the image of the induced map $\mathbb{F}^{e}(N) \rightarrow \mathbb{F}^{e}(M)$.

Definition 1.1. Let $\mathfrak{a}$ be an ideal of a Noetherian ring $R$ of characteristic $p>0$ and let $N \subseteq M$ be $R$-modules. The a-tight closure of $N$ in $M$, denoted by $N_{M}^{* \mathfrak{a}}$, is defined to be the submodule of $M$ consisting of all elements $z \in M$ for which there exists $c \in R^{\circ}$ such that

$$
c z^{q} \mathfrak{a}^{q} \subseteq N_{M}^{[q]}
$$

for all large $q=p^{e}$. The a-tight closure of an ideal $I \subseteq R$ is just defined by $I^{* \mathfrak{a}}=I_{R}^{* \mathfrak{a}}$.

Remark 1.2. (1) In the case where $\mathfrak{a}=R$ is the unit ideal, the a-tight closure $N_{M}^{* \mathfrak{a}}=N_{M}^{* R}$ is nothing but the (usual) tight closure $N_{M}^{*}$ as defined in [HH1]. However, unlike the usual tight closure, it may happen that $\left(N_{M}^{* \mathfrak{a}}\right)_{M}^{* \mathfrak{a}}$ is strictly larger than $N_{M}^{* \mathfrak{a}}$; see Remark 1.4 (1). In this sense $\mathfrak{a}$-tight closure is not an "honest" closure operation in general. 
(2) It seems significant to extend the definition to "rational coefficients", if we take into account the relationship with multiplier ideals; see [La], [T] and Sections 3 and 4. Namely, given nonnegative $t \in \mathbb{Q}$ and $\mathfrak{a} \subseteq R, N \subseteq M$ as in Definition 1.1, we can define the $t \cdot \mathfrak{a}$-tight closure $N_{M}^{* t \cdot \mathfrak{a}}$ of $N$ in $M$ and generalize some results for $\mathfrak{a}$-tight closure to those for $t \cdot \mathfrak{a}$-tight closure. We treat this issue in Section 6 .

We collect some basic properties of $\mathfrak{a}$-tight closure in the following. The proofs are easy and are left to the reader.

Proposition 1.3. Let $\mathfrak{a}$ and $\mathfrak{b}$ denote ideals of a Noetherian ring $R$ of characteristic $p>0$ and let $L$ and $N$ denote submodules of an $R$-module $M$.

(1) $N \subseteq N_{M}^{* \mathfrak{a}}$ and $N_{M}^{* \mathfrak{a}} / N \cong 0_{M / N}^{* \mathfrak{a}}$.

(2) If $L \subseteq N$, then $L_{M}^{* \mathfrak{a}} \subseteq N_{M}^{* \mathfrak{a}}$.

(3) $N_{M}^{* \mathfrak{a} \mathfrak{b}} \subseteq\left(N_{M}^{* \mathfrak{a}}: \mathfrak{b}\right)_{M}$. Moreover, if $\mathfrak{b}$ is a principal ideal, then the equality $N_{M}^{* \mathfrak{a} \mathfrak{b}}=\left(N_{M}^{* \mathfrak{a}}: \mathfrak{b}\right)_{M}$ holds.

(4) If $\mathfrak{b} \subseteq \mathfrak{a}$, then $N_{M}^{* \mathfrak{a}} \subseteq N_{M}^{* \mathfrak{b}}$. Moreover, if $\mathfrak{a} \cap R^{\circ} \neq \emptyset$ and if $\mathfrak{b}$ is a reduction of $\mathfrak{a}$, then the equality $N_{M}^{* \mathfrak{a}}=N_{M}^{* \mathfrak{b}}$ holds.

Remark 1.4. (1) It follows that $N_{M}^{*} \subseteq N_{M}^{* \mathfrak{a}} \subseteq\left(N_{M}^{*}: \mathfrak{a}\right)_{M}$ from (3) and (4) of Proposition 1.3. If $\mathfrak{a}$ is a principal ideal, then the equality on the right occurs, and $\left(N_{M}^{* \mathfrak{a}}\right)_{M}^{* \mathfrak{a}}=\left(N_{M}^{*}: \mathfrak{a}^{2}\right)_{M}$ is strictly larger than $N_{M}^{* \mathfrak{a}}=\left(N_{M}^{*}: \mathfrak{a}\right)_{M}$ in general.

(2) The colon-capturing property [HH1, Section 7] says that parameters behave like a regular sequence modulo tight closure. Namely, if $x_{1}, \ldots, x_{i+1} \in R$ are parameters, then under a mild assumption, $\left(x_{1}, \ldots, x_{i}\right):_{R} x_{i+1} \subseteq\left(x_{1}, \ldots, x_{i}\right)^{*}$. Since $\mathfrak{a}$-tight closure contains the usual tight closure, this remains true if we replace the usual tight closure by a-tight closure. In Proposition 1.5 below we slightly improve this colon-capturing property for $\mathfrak{a}$-tight closure using the existence of a test element. See [HH1], [HH2] for the definition and detailed study of test elements, and see also Definition 1.6 for a generalization to the notion of a-test elements.

Proposition 1.5. Let $R$ be an equidimensional reduced excellent ring of characteristic $p>0$ and let $\mathfrak{a}$ be an ideal. Then for any parameters $x_{1}, \ldots, x_{n}$ in $R$,

$$
\left(x_{1}, \ldots, x_{n-1}\right)^{* \mathfrak{a}}: x_{n} \subseteq\left(x_{1}, \ldots, x_{n-1}\right)^{* \mathfrak{a}} .
$$

Proof. Actually, we can prove even more. Namely, let $I, J \subseteq R$ be ideals generated by monomials in parameters $x_{1}, \ldots, x_{n}$, and let $K \subseteq R$ be the "expected" answer for $I: J$, that is, the monomial ideal that would be equal to $I: J$ if $x_{1}, \ldots, x_{n}$ formed a regular sequence. (Note that $I=K=\left(x_{1}, \ldots, x_{n-1}\right)$ and $J=\left(x_{n}\right)$ in our case.) Then we will show that

$$
I^{* \mathfrak{a}}: J \subseteq K^{* \mathfrak{a}} \text {. }
$$

Let $z \in I^{* \mathfrak{a}}: J$. Then there is a $c \in R^{\circ}$ such that $c z^{q} \mathfrak{a}^{q} \subseteq I^{[q]}: J^{[q]}$ for $q=p^{e} \gg 0$. Since the "expected" answer for $I^{[q]}: J^{[q]}$ is $K^{[q]}$, we have that $I^{[q]}: J^{[q]} \subseteq\left(K^{[q]}\right)^{*}$ by colon-capturing of the usual tight closure [HH1]. So, for a test element $d \in R^{\circ}$, one has that $(c d) z^{q} \mathfrak{a}^{q} \subseteq K^{[q]}$ for $q=p^{e} \gg 0$, which means that $z \in K^{* \mathfrak{a}}$, as required.

Definition 1.6. Let $R$ be a Noetherian ring of characteristic $p>0$ and let $\mathfrak{a}$ be an ideal of $R$. We say that an element $c \in R^{\circ}$ is an $\mathfrak{a}$-test element if $c z^{q} \mathfrak{a}^{q} \subseteq I^{[q]}$ for all $q=p^{e}$ whenever $z \in I^{* \mathfrak{a}}$. 
In the case where $\mathfrak{a}=R$ is the unit ideal, an $\mathfrak{a}$-test element is nothing but a test element in the usual sense [HH1]. In [HH2] it is proved that a test element exists in nearly every ring of interest, for example, in excellent reduced local rings [HH2. Theorem 6.1]. We can show that an a-test element also does.

Theorem 1.7. Let $R$ be a reduced Noetherian ring of characteristic $p>0$, let $c \in R^{\circ}$, and assume that one of the following conditions holds:

(1) $R$ is F-finite and the localized ring $R_{c}$ is strongly F-regular;

(2) $R$ is an excellent local ring and $R_{c}$ is Gorenstein and F-regular.

Then some power $c^{n}$ of $c$ is an $\mathfrak{a}$-test element for all ideals $\mathfrak{a} \subseteq R$.

Here we prove the above theorem under assumption (1) only, according to the method of [HH0]. The case of assumption (2) is reduced to the F-finite case by the machinery of " $\Gamma$-construction" used in [HH2]. We do not include the argument involving this reduction process, because it is somewhat long but essentially the same as that for the usual tight closure [HH2, Section 6].

To prove the theorem in the F-finite case we need the following lemma from [HH0. Remark 3.2], in which it is implicit that the exponent $n$ of $c$ may be independent of the choice of $d$.

Lemma 1.8. Let $R$ be an F-finite reduced Noetherian ring of characteristic $p>0$. If the localization $R_{c}$ of $R$ at an element $c \in R^{\circ}$ is strongly F-regular, then there exists an integer $n \geq 0$, depending only on $R$ and $c$, satisfying the following property: For any $d \in R^{\circ}$, there exist a power $q^{\prime}$ of $p$ and an $R$-linear map $\phi: R^{1 / q^{\prime}} \rightarrow R$ sending $d^{1 / q^{\prime}}$ to $c^{n}$.

Proof of Theorem 1.7 in case (1). We will show that $c^{n}$ in Lemma 1.8 is an a-test element for every $\mathfrak{a} \subseteq R$. Given any ideal $I$, any $z \in I^{* \mathfrak{a}}$ and any power $q$ of $p$, it is enough to show that $c^{n} z^{q} \mathfrak{a}^{q} \subseteq I^{[q]}$. Since $z \in I^{* \mathfrak{a}}$, there exists $d \in R^{\circ}$ such that $d z^{Q} \mathfrak{a}^{Q} \subseteq I^{[Q]}$ for every $Q$. Then by Lemma 1.8 , there exist $q^{\prime}$ and $\phi: R^{1 / q^{\prime}} \rightarrow R$ sending $d^{1 / q^{\prime}}$ to $c^{n}$. Since $d z^{q q^{\prime}}\left(\mathfrak{a}^{q}\right)^{\left[q^{\prime}\right]} \subseteq d z^{q q^{\prime}} \mathfrak{a}^{q q^{\prime}} \subseteq I^{\left[q q^{\prime}\right]}$, one has

$$
d^{1 / q^{\prime}} z^{q} \mathfrak{a}^{q} R^{1 / q^{\prime}} \subseteq I^{[q]} R^{1 / q^{\prime}},
$$

and applying $\phi$ gives $c^{n} z^{q} \mathfrak{a}^{q} \subseteq I^{[q]}$, as required.

Proposition-Definition 1.9. Let $R$ be a Noetherian ring of characteristic $p>0$ and let $\mathfrak{a}$ be an ideal of $R$. Let $E=\bigoplus_{\mathfrak{m}} E_{R}(R / \mathfrak{m})$, the direct sum, taken over all maximal ideals $\mathfrak{m}$ of $R$, of the injective envelopes of the residue fields $R / \mathfrak{m}$. Then the following ideals are equal to each other, and we denote them by $\tau(\mathfrak{a})$ :

i) $\bigcap \operatorname{Ann}_{R}\left(0_{M}^{* \mathfrak{a}}\right)$, where $M$ runs through all finitely generated $R$-modules;

ii) $\bigcap_{M \subseteq E}^{M} \operatorname{Ann}_{R}\left(0_{M}^{* \mathfrak{a}}\right)$, where $M$ runs through all finitely generated $R$-submodules of $E$.

If $R$ is locally approximately Gorenstein (e.g., if $R$ is excellent and reduced [Ho1]), then the following ideal is also equal to $\tau(\mathfrak{a})$ :

iii) $\bigcap_{I \subseteq R}\left(I: I^{* \mathfrak{a}}\right)$, where $I$ runs through all ideals of $R$.

Proof. The proof is the same as that for the usual tight closure. See [HH1, Proposition 8.23] for the equality of i) and ii), and [HH1, Proposition 8.25] for the equality of ii) and iii). 
Remark 1.10. In the case where $\mathfrak{a}=R$ is the unit ideal, $\tau(\mathfrak{a})=\tau(R)$ is called the test ideal. In this case, $\tau(R) \cap R^{\circ}$ is equal to the set of test elements of $R$, and this justifies the name "test ideal". But the name "a-test ideal" for $\tau(\mathfrak{a})$ is somewhat misleading if $\mathfrak{a} \neq R$, because $\tau(\mathfrak{a}) \cap R^{\circ}$ is not equal to the set of $\mathfrak{a}$-test elements in general.

The following basic properties of the ideal $\tau(\mathfrak{a})$ follow from Proposition 1.3. See Theorem 2.1 for a generalization of the latter half of (1).

Proposition 1.11. Let $R$ be a Noetherian ring of characteristic $p>0$ and let $\mathfrak{a}$ and $\mathfrak{b}$ denote ideals of $R$.

(1) $\tau(\mathfrak{a}) \mathfrak{b} \subseteq \tau(\mathfrak{a} \mathfrak{b})$. Moreover, if $\mathfrak{b}$ is a principal ideal of a complete local ring, then $\tau(\mathfrak{a}) \mathfrak{b}=\tau(\mathfrak{a} \mathfrak{b})$.

(2) If $\mathfrak{b} \subseteq \mathfrak{a}$, then $\tau(\mathfrak{b}) \subseteq \tau(\mathfrak{a})$. Moreover, if $\mathfrak{a} \cap R^{\circ} \neq \emptyset$ and if $\mathfrak{b}$ is a reduction of $\mathfrak{a}$, then $\tau(\mathfrak{b})=\tau(\mathfrak{a})$.

(3) If $R$ admits a test element and if $\mathfrak{a} \cap R^{\circ} \neq \emptyset$, then $\tau(\mathfrak{a}) \cap R^{\circ} \neq \emptyset$.

(4) If $R$ is weakly F-regular, then $\mathfrak{a} \subseteq \tau(\mathfrak{a})$. Moreover, if $\mathfrak{a}$ is an ideal of pure height one, then $\mathfrak{a}=\tau(\mathfrak{a})$.

Proof. The first half of (1) is immediate from Proposition 1.3 (3). To prove the second half, let $(R, \mathfrak{m})$ be a complete local ring and let $\mathfrak{b}$ be principal. Then by the Matlis duality, $\operatorname{Ann}_{E}(\tau(\mathfrak{a}))$ is equal to the union of $0_{M}^{* \mathfrak{a}}$ taken over all finitely generated submodules $M$ of $E=E_{R}(R / \mathfrak{m})$. So, if $z \in \operatorname{Ann}_{E}(\tau(\mathfrak{a}) \mathfrak{b})$, then there exists a finitely generated submodule $M \subset E$ such that $z \in\left(0_{M}^{* \mathfrak{a}}: \mathfrak{b}\right)_{E}$. Replacing $M$ by $M+R z \subset E$, one has $z \in\left(0_{M}^{* \mathfrak{a}}: \mathfrak{b}\right)_{M}=0_{M}^{* \mathfrak{a} \mathfrak{b}}$ by Proposition 1.3 (3). Hence

$$
\tau(\mathfrak{a} \mathfrak{b})=\bigcap_{M \subseteq E} \operatorname{Ann}_{R}\left(0_{M}^{* \mathfrak{a}}: \mathfrak{b}\right)_{M}=\operatorname{Ann}_{R}\left(\operatorname{Ann}_{E}(\tau(\mathfrak{a}) \mathfrak{b})\right)=\tau(\mathfrak{a}) \mathfrak{b}
$$

(2) follows from Proposition 1.3 (4), and (3) and the first half of (4) from $\tau(R) \mathfrak{a} \subseteq$ $\tau(\mathfrak{a})$. As for the second half of (4), it suffices to show the following claim, since weakly F-regular rings are normal.

Claim 1.11.1. If $R$ is normal and $\mathfrak{a}$ is an ideal of pure height one, then $\tau(\mathfrak{a}) \subseteq \mathfrak{a}$.

To prove the claim, considering a primary decomposition of $\mathfrak{a}$, we may assume, without loss of generality, that $\mathfrak{a}$ is a primary ideal such that $\mathfrak{p}=\sqrt{\mathfrak{a}}$ is a height one prime ideal. Then, since $R_{\mathfrak{p}}$ is a discrete valuation ring, we can choose $b \in \mathfrak{a}$ such that $\mathfrak{a} R_{\mathfrak{p}}=b R_{\mathfrak{p}}$. Then $b R: \mathfrak{a} \subseteq \mathfrak{a}^{* \mathfrak{a}}$. Indeed, if $z \in b R: \mathfrak{a}$, then $z^{q} \mathfrak{a}^{q} \subseteq b^{q} R \subseteq \mathfrak{a}^{[q]}$

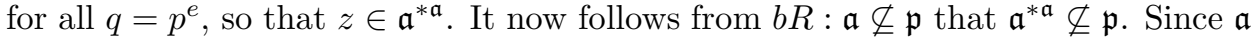
is $\mathfrak{p}$-primary, we have $\tau(\mathfrak{a}) \subseteq \mathfrak{a}: \mathfrak{a}^{* \mathfrak{a}}=\mathfrak{a}$, as claimed.

Proposition 1.12 (cf. [B], [HH1, Proposition 4.12]). Let $R \subseteq S$ be a pure ring extension of Noetherian rings of characteristic $p>0$ such that $R^{\circ} \subseteq S^{\circ}$. Then for any ideal $\mathfrak{a}$ of $R$, one has $\tau(\mathfrak{a} S) \cap R \subseteq \tau(\mathfrak{a})$.

Proof. For a finitely generated $R$-module $M$, the natural map $M=M \otimes_{R} R \rightarrow$ $M \otimes_{R} S$ is injective by the purity of $R \subseteq S$. Since $R^{\circ} \subseteq S^{\circ}$, we see easily that $0_{M}^{* \mathfrak{a}} \subseteq 0_{M \otimes S}^{* \mathfrak{a} S}$ via the inclusion map $M \hookrightarrow M \otimes_{R} S$. Hence, if $c \in \tau(\mathfrak{a} S) \cap R$, then $c$ kills $0_{M}^{* \mathfrak{a}}$ for all finitely generated $R$-modules $M$, so that $c \in \tau(\mathfrak{a})$.

By definition, the ideal $\tau(\mathfrak{a})$ is the annihilator of $\mathfrak{a}$-tight closure relations for all ideals or finitely generated modules. It will be very useful if $\tau(\mathfrak{a})$ is determined by 
a-tight closure relations for a single ideal or a single module. Let us take a look at some cases where this is true.

Theorem 1.13. Let $(R, \mathfrak{m})$ be a d-dimensional excellent normal local ring of characteristic $p>0, \mathfrak{a}$ an ideal of $R$, and let $J \subseteq R$ be a divisorial ideal such that the divisor class $\mathrm{cl}(J) \in \mathrm{Cl}(R)$ has a finite order. Then

$$
0_{H_{\mathfrak{m}}^{d}(J)}^{* \mathfrak{a}}=\bigcup_{M \subset H_{\mathfrak{m}}^{d}(J)} 0_{M}^{* \mathfrak{a}},
$$

where $M$ runs through all finitely generated $R$-submodules of $H_{\mathfrak{m}}^{d}(J)$. In particular, if $R$ is $\mathbb{Q}$-Gorenstein, then

$$
\tau(\mathfrak{a})=\operatorname{Ann}_{R}\left(0_{E}^{* \mathfrak{a}}\right)
$$

where $E=E_{R}(R / \mathfrak{m}) \cong H_{\mathfrak{m}}^{d}\left(\omega_{R}\right)$.

Proof. Again the proof is the same as that for the usual tight closure 1 but we sketch a proof according to Sm2, Lemma 3.4], which is based on the idea of [AM, $3.1]$.

Let $r$ be the order of $\operatorname{cl}(J) \in \mathrm{Cl}(R)$ and let $J^{(r)}=x_{1} R$. We may assume, without loss of generality, that $x_{1} \in \mathfrak{m}$. Then there exist $x_{2} \in R$ and $0 \neq a \in J$ such that $x_{2} J \subseteq a R$, and $x_{1}, x_{2}$ extends to a system of parameters $x_{1}, x_{2}, \ldots, x_{d}$ for $R$.

The point of the proof is that $\mathbb{F}^{e}\left(H_{\mathfrak{m}}^{d}(J)\right) \cong H_{\mathfrak{m}}^{d}\left(J^{\left(p^{e}\right)}\right)$ is computed by

$$
H_{\mathfrak{m}}^{d}\left(J^{(q)}\right)=\lim _{\rightarrow} R /\left(x_{1}^{s} J^{(q)}, x_{2}^{s}, \ldots, x_{d}^{s}\right),
$$

where the direct limit map $R /\left(x_{1}^{s} J^{(q)}, x_{2}^{s}, \ldots, x_{d}^{s}\right) \rightarrow R /\left(x_{1}^{s+1} J^{(q)}, x_{2}^{s+1}, \ldots, x_{d}^{s+1}\right)$ is the multiplication by $x_{1} x_{2} \cdots x_{d}$. Then an element $\xi \in H_{\mathfrak{m}}^{d}(J)$ is represented by $z \bmod \left(x_{1}^{s} J, x_{2}^{s}, \ldots, x_{d}^{s}\right) \in R /\left(x_{1}^{s} J, x_{2}^{s}, \ldots, x_{d}^{s}\right)$ for some $z \in R$ and $s \in \mathbb{N}$, and $\xi=$ $\left[z \bmod \left(x_{1}^{s} J, x_{2}^{s}, \ldots, x_{d}^{s}\right)\right]$ is mapped to $\xi^{p^{e}}=\left[z^{p^{e}} \bmod \left(x_{1}^{p^{e} s} J^{\left(p^{e}\right)}, x_{2}^{p^{e}}, \ldots, x_{d}^{p^{e} s}\right)\right]$ by the $e$-times iterated Frobenius map $F^{e}: H_{\mathfrak{m}}^{d}(J) \rightarrow H_{\mathfrak{m}}^{d}\left(J^{\left(p^{e}\right)}\right)$.

Now say that $\xi \in 0_{H_{\mathrm{m}}^{d}(J)}^{* \mathfrak{a}}$. Then there exists $c \in R^{\circ}$ such that $c \xi^{q} \alpha=\left[c z^{q} \alpha \bmod \right.$ $\left.\left(x_{1}^{q s} J^{(q)}, x_{2}^{q s}, \ldots, x_{d}^{q s}\right)\right]=0$ for all $q=p^{e} \gg 0$ and $\alpha \in \mathfrak{a}^{q}$. Since $\mathfrak{a}^{q}$ is a finitely generated ideal for each $q=p^{e}$, there exists $t_{e} \in \mathbb{N}$ such that $c z^{q}\left(x_{1} \cdots x_{d}\right)^{t_{e}} \mathfrak{a}^{q} \subseteq$ $\left(x_{1}^{q s+t_{e}} J^{(q)}, x_{2}^{q s+t_{e}}, \ldots, x_{d}^{q s+t_{e}}\right) \subseteq\left(x_{1}^{q s+t_{e}+\lfloor q / r\rfloor}, x_{2}^{q s+t_{e}}, \ldots, x_{d}^{q s+t_{e}}\right)$. Then one has $c z^{q} \mathfrak{a}^{q} \subseteq\left(x_{1}^{q s+\lfloor q / r\rfloor}, x_{2}^{q s}, \ldots, x_{d}^{q s}\right)^{*}$ by colon-capturing. Replacing $c$ by $c c^{\prime}$ with $c^{\prime}$ a test element and multiplying by $x_{1}$, we see that $c x_{1} z^{q} \mathfrak{a}^{q} \subseteq\left(x_{1}^{q s} J^{(q)}, x_{2}^{q s}, \ldots, x_{d}^{q s}\right)$. This gives

$$
c x_{1}\left(x_{1} \cdots x_{d}\right)^{q} z^{q} \mathfrak{a}^{q} \subseteq\left(x_{1}^{q(s+1)} a^{q}, x_{2}^{q(s+1)}, \ldots, x_{d}^{q(s+1)}\right) \subseteq\left(x_{1}^{s+1} J, x_{2}^{s+1}, \ldots, x_{d}^{s+1}\right)^{[q]}
$$

for all $q=p^{e} \gg 0$, whence $\left(x_{1} \cdots x_{d}\right) z \in\left(x_{1}^{s+1} J, x_{2}^{s+1}, \ldots, x_{d}^{s+1}\right)^{* \mathfrak{a}}$. Hence $\xi$ is in the $\mathfrak{a}$-tight closure of zero in the cyclic (hence finitely generated) submodule of $H_{\mathfrak{m}}^{d}(J)$ generated by the image of $R /\left(x_{1}^{s+1} J, x_{2}^{s+1}, \ldots, x_{d}^{s+1}\right)$.

Discussion 1.14. In Sections 2 and 5, we will consider when the equality $\tau(\mathfrak{a})=R$ holds. When $R$ is a Gorenstein local ring, one can check this condition looking only at the $\mathfrak{a}$-tight closure of a single parameter ideal, as we will see below; cf. [FW].

Let $(R, \mathfrak{m})$ be a $d$-dimensional Cohen-Macaulay local ring, $\mathfrak{a}$ any ideal of $R$, and let $J$ be the ideal generated by a system of parameters $x_{1}, \ldots, x_{d}$. Then

1 The proof in [Ha2, Appendix] has a minor gap at the bottom of p. 1904, although the result [Ha2 Theorem 1.8] itself and the arguments in the cited references [Mc , Wi are valid. 
$H_{\mathfrak{m}}^{d}(R) \cong \lim _{\rightarrow} R /\left(x_{1}^{t}, \ldots, x_{d}^{t}\right)$, and $R / J$ and $H_{\mathfrak{m}}^{d}(R)$ have the same socle in common via the natural inclusion map $R / J \hookrightarrow H_{\mathfrak{m}}^{d}(R)$. Then $0_{H_{\mathfrak{m}}^{d}(R)}^{* \mathfrak{a}}=0$ if and only if $0_{R / J}^{* \mathfrak{a}}=0$ or, equivalently, if $J^{* \mathfrak{a}}=J$. In particular, the condition that $J$ is $\mathfrak{a}$-tightly closed does not depend on the choice of a parameter ideal $J$.

Now assume further that $(R, \mathfrak{m})$ is Gorenstein. Then $E=E_{R}(R / \mathfrak{m}) \cong H_{\mathfrak{m}}^{d}(R)$, and one sees easily that $\tau(\mathfrak{a})=\operatorname{Ann}_{R}\left(0_{H_{\mathfrak{m}}^{d}(R)}^{* \mathfrak{a}}\right)=\bigcap_{t \in \mathbb{N}}\left(x_{1}^{t}, \ldots, x_{d}^{t}\right):\left(x_{1}^{t}, \ldots, x_{d}^{t}\right)^{* \mathfrak{a}}$. Therefore $\tau(\mathfrak{a})=R$ if and only if $J^{* \mathfrak{a}}=J$ for some (or equivalently, every) ideal $J$ generated by a system of parameters.

As we have seen so far, the $\mathfrak{a}$-tight closure of the zero submodule in the injective envelope $E_{R}(R / \mathfrak{m})$ or the top local cohomology $H_{\mathfrak{m}}^{d}(R)$ of a local ring $(R, \mathfrak{m})$ plays a particularly important role. We close this section by the following proposition, which generalizes Smith's characterization of the usual tight closure of the zero submodule in $H_{\mathfrak{m}}^{d}(R)$.

Proposition 1.15 (cf. [Sm1]). Let $(R, \mathfrak{m})$ be a d-dimensional excellent normal local ring of characteristic $p>0$ and let $\mathfrak{a} \subseteq R$ be an ideal such that $\mathfrak{a} \cap R^{\circ} \neq \emptyset$. Then $0_{H_{\mathrm{m}}^{d}(R)}^{* \mathfrak{a}}$ is the unique maximal proper submodule $N$ with respect to the property

$$
\mathfrak{a}^{q} F^{e}(N) \subseteq N \text { for all } q=p^{e},
$$

where $F^{e}: H_{\mathfrak{m}}^{d}(R) \rightarrow H_{\mathfrak{m}}^{d}(R)$ is the e-times iterated Frobenius induced on $H_{\mathfrak{m}}^{d}(R)$.

Proof. Let $c \in R^{\circ}$ be an element such that $R_{c}$ is regular. Then $\hat{R}_{c}$ is also regular by the excellence of $R$. Hence some power $c^{n}$ of $c$ is an $\mathfrak{a}$-test element and an $\mathfrak{a} \hat{R}$-test element, by Theorem 1.7. It is easy to see that $c^{n}$ also works as a test element for both the $\mathfrak{a}$-tight closure and the $\mathfrak{a} \hat{R}$-tight closure of the zero submodule in $H_{\mathfrak{m}}^{d}(R)=$ $H_{\mathfrak{m} \hat{R}}^{d}(\hat{R})$ (see the proof of Theorem 1.13). Then it follows that $0_{H_{\mathfrak{m}}^{d}(R)}^{* \mathfrak{a}}=0_{H_{\mathfrak{m} \hat{R}}^{d}(\hat{R})}^{* \hat{R}} ;$ so we may assume, without loss of generality, that $R$ is a complete local ring.

It is easy to see that $\mathfrak{a}^{q} F^{e}\left(0_{H_{\mathfrak{m}}^{d}(R)}^{* \mathfrak{a}}\right) \subseteq 0_{H_{\mathfrak{m}}^{d}(R)}^{* \mathfrak{a}}$ for all $q=p^{e}$. Also, $0_{H_{\mathfrak{m}}^{d}(R)}^{* \mathfrak{a}}$ is a proper submodule of $H_{\mathfrak{m}}^{d}(R)$, because it is annihilated by $\tau(\mathfrak{a})$ by Theorem 1.13 and $\tau(\mathfrak{a}) \cap R^{\circ} \neq \emptyset$ by Proposition $1.11(3)$. To prove the maximality of $0_{H_{\mathfrak{m}}^{d}}^{* \mathfrak{a}}(R)$, suppose that $N \subset H_{\mathfrak{m}}^{d}(R)$ is a proper submodule such that $\mathfrak{a}^{q} F^{e}(N) \subseteq N$ for all $q=p^{e}$. Then the Matlis dual of the exact sequence $0 \rightarrow N \rightarrow H_{\mathfrak{m}}^{d}(R) \rightarrow H_{\mathfrak{m}}^{d}(R) / N \rightarrow 0$ is

$$
0 \rightarrow\left[H_{\mathfrak{m}}^{d}(R) / N\right]^{\vee} \rightarrow \omega_{R} \rightarrow N^{\vee} \rightarrow 0,
$$

where $\left[H_{\mathfrak{m}}^{d}(R) / N\right]^{\vee}$ is a nonzero submodule of $\omega_{R}$. So both $\left[H_{\mathfrak{m}}^{d}(R) / N\right]^{\vee}$ and $\omega_{R}$ are torsion-free $R$-modules of rank 1. Therefore $N^{\vee}$ is a finitely generated torsion module, so that there exists $c \in R^{\circ}$ such that $c N^{\vee}=0$. This implies that $c N=$ $c N^{\vee \vee}=0$, so that $c \mathfrak{a}^{q} F^{e}(N)=0$ for all $q=p^{e}$. Hence $N \subseteq 0_{H_{\mathfrak{m}}^{d}(R)}^{* \mathfrak{d}}$, as required.

\section{2. a-Tight ClOSURE AND ITS APPLiCATIONS}

In this section we give some fundamental applications of $\mathfrak{a}$-tight closure.

Modified Briançon-Skoda theorem via a-tight closure. One of the important applications of tight closure theory [HH1] is a prime characteristic proof of the Briançon-Skoda theorem $\overline{B S}$, which was originally proved by an analytic method. Later, Lipman [Li] improved this in terms of adjoint ideals. The following is a prime characteristic analog of Lipman's "modified Briançon-Skoda" [Li, Theorem 1.4.1]; see also Remark $3.2(1)$. 
Theorem 2.1. Let $R$ be a Noetherian ring of characteristic $p>0$. If $\mathfrak{a} \subseteq R$ is an ideal generated by $r$ elements, then

$$
\tau\left(\mathfrak{a}^{n+r-1}\right) \subseteq \mathfrak{a}^{n}
$$

for all $n \geq 0$. If we assume further that $R$ is a complete local ring, then

$$
\tau\left(\mathfrak{a}^{n+r-1} \mathfrak{b}\right) \subseteq \tau(\mathfrak{b}) \mathfrak{a}^{n}
$$

for all $n \geq 0$ and all ideals $\mathfrak{b} \subseteq R$.

Proof. Let $\mathfrak{b} \subseteq R$ be any ideal, $M$ any finitely generated $R$-module, and suppose that $z \in\left(0_{M}^{* \mathfrak{b}}: \mathfrak{a}^{n}\right)_{M}$. Then there exists $c \in R^{\circ}$ such that $c z^{q}\left(\mathfrak{a}^{n}\right)^{[q]} \mathfrak{b}^{q}=0$ in $\mathbb{F}^{e}(M)$ for all $q=p^{e} \gg 0$. Since $\mathfrak{a}^{(n+r-1) q} \subseteq\left(\mathfrak{a}^{n}\right)^{[q]}$ by the assumption, this implies that $c z^{q}\left(\mathfrak{a}^{n+r-1} \mathfrak{b}\right)^{q}=0$ in $\mathbb{F}^{e}(M)$ for all $q=p^{e} \gg 0$, so that $z \in 0_{M}^{* \mathfrak{a}^{n+r-1} \mathfrak{b}}$. Thus $\left(0_{M}^{* \mathfrak{b}}: \mathfrak{a}^{n}\right)_{M} \subseteq 0_{M}^{* \mathfrak{a}^{n+r-1} \mathfrak{b}}$ and, in particular, $\operatorname{Ann}_{M}\left(\mathfrak{a}^{n}\right) \subseteq 0_{M}^{* \mathfrak{a}^{n+r-1}}$. Taking the intersection of the annihilator ideals over all finitely generated $R$-submodules $M \subseteq E=\bigoplus_{\mathfrak{m}} E_{R}(R / \mathfrak{m})$, we obtain

$$
\tau\left(\mathfrak{a}^{n+r-1}\right) \subseteq \bigcap_{M \subseteq E} \operatorname{Ann}_{R}\left(\operatorname{Ann}_{M}\left(\mathfrak{a}^{n}\right)\right)=\operatorname{Ann}_{R}\left(\operatorname{Ann}_{E}\left(\mathfrak{a}^{n}\right)\right)=\mathfrak{a}^{n} .
$$

Now assume that $(R, \mathfrak{m})$ is a complete local ring. $\operatorname{Then} \operatorname{Ann}_{E}(\tau(\mathfrak{b}))=\bigcup_{M \subset E} 0_{M}^{* \mathfrak{b}}$ by the Matlis duality, and it follows as in the latter half of Proposition 1.11 (1) that $\operatorname{Ann}_{E}\left(\tau(\mathfrak{b}) \mathfrak{a}^{n}\right) \subseteq \bigcup_{M \subseteq E}\left(0_{M}^{* \mathfrak{b}}: \mathfrak{a}^{n}\right)_{M} \subseteq \bigcup_{M \subseteq E} 0_{M}^{* \mathfrak{a}^{n+r-1} \mathfrak{b}}$, where the unions are taken over all finitely generated submodules $M$ of $E$. Thus we conclude that

$$
\tau\left(\mathfrak{a}^{n+r-1} \mathfrak{b}\right) \subseteq \bigcap_{M \subseteq E} \operatorname{Ann}_{R}\left(0_{M}^{* \mathfrak{b}}: \mathfrak{a}^{n}\right)_{M}=\operatorname{Ann}_{R}\left(\operatorname{Ann}_{E}\left(\tau(\mathfrak{b}) \mathfrak{a}^{n}\right)\right)=\tau(\mathfrak{b}) \mathfrak{a}^{n} .
$$

Remark 2.2. The tight closure version of the Briançon-Skoda theorem [HH1, Theorem 5.4] says that if $\mathfrak{a}$ is generated by $r$ elements, then $\overline{\mathfrak{a}^{n+r-1}} \subseteq\left(\mathfrak{a}^{n}\right)^{*}$ for all $n \geq 0$, where $\overline{\mathfrak{b}}$ denotes the integral closure of an ideal $\mathfrak{b}$. This implies that $\tau(R) \overline{\mathfrak{a}^{n+r-1}} \subseteq \mathfrak{a}^{n}$, and in the case where the test ideal $\tau(R)$ is a strong test ideal (this is the case if $R$ is a reduced complete local ring $[\mathrm{Vr}]$ ), $\tau(R) \overline{\mathfrak{a}^{n+r-1}} \subseteq \tau(R) \mathfrak{a}^{n}$. Theorem 2.1 may be considered a slight improvement of these assertions, because $\tau(R) \overline{\mathfrak{a}^{n+r-1}} \subseteq \tau\left(\overline{\mathfrak{a}^{n+r-1}}\right)=\tau\left(\mathfrak{a}^{n+r-1}\right)$ by basic properties of $\mathfrak{a}$-tight closure; see also Discussion 5.2.

Recently, using arguments similar to the above, the first author and S. Takagi proved a sharpened version of Theorem $2.1[\mathrm{HT}]$; $\mathrm{cf}$. [Li], [La]: if $(R, \mathfrak{m})$ is a complete local ring of characteristic $p>0$ and if $\mathfrak{a}$ is an ideal with a reduction generated by $r$ elements, then $\tau\left(\mathfrak{a}^{n+r-1}\right)=\tau\left(\mathfrak{a}^{r-1}\right) \mathfrak{a}^{n}$ for all $n \geq 0$.

Corollary 2.3. Let $R$ be a reduced excellent ring of characteristic $p>0$ and let $\mathfrak{a}$ be an ideal such that $\mathfrak{a} \cap R^{\circ} \neq \emptyset$. Then for any $R$-modules $N \subset M$ and any $z \in M$, the following conditions are equivalent.

(1) $z \in N_{M}^{* \mathfrak{a}}$, i.e., there exists $c \in R^{\circ}$ such that $c z^{q} \mathfrak{a}^{q} \subseteq N_{M}^{[q]}$ for all $q=p^{e}$.

(2) There exists $c \in R^{\circ}$ such that $c z^{q} \tau\left(\mathfrak{a}^{q}\right) \subseteq N_{M}^{[q]}$ for all $q=p^{e}$.

(3) There exists $c \in R^{\circ}$ such that $c z^{q} \overline{\mathfrak{a}^{q}} \subseteq N_{M}^{[q]}$ for all $q=p^{e}$.

Proof. To prove $(1) \Rightarrow(2)$, choose $d \in \mathfrak{a}^{r-1} \cap R^{\circ}$ and apply $d \tau\left(\mathfrak{a}^{q}\right) \subseteq \tau\left(\mathfrak{a}^{q+r-1}\right) \subseteq \mathfrak{a}^{q}$. As for $(2) \Rightarrow(3)$, choose a test element $d \in \tau(R) \cap R^{\circ}$ and note that $d \overline{\mathfrak{a}^{q}} \subseteq \tau\left(\mathfrak{a}^{q}\right)$. 
Corollary 2.4. Let $(R, \mathfrak{m})$ be a d-dimensional Noetherian local ring of characteristic $p>0$ with infinite residue field. Then for any ideal $\mathfrak{a} \subseteq R$ and for any $n>0$, one has $\tau\left(\mathfrak{a}^{n+d-1}\right) \subseteq \mathfrak{a}^{n}$. If, in addition, $(R, \mathfrak{m})$ is complete, then $\tau\left(\mathfrak{a}^{n+d-1}\right) \subseteq \tau(R) \mathfrak{a}^{n}$.

Proof. First assume that $\mathfrak{a}$ is an $\mathfrak{m}$-primary ideal. Then $\mathfrak{a}$ has a minimal reduction $\mathfrak{q} \subseteq \mathfrak{a}$ generated by $d$ elements, so that

$$
\tau\left(\mathfrak{a}^{n+d-1}\right)=\tau\left(\mathfrak{q}^{n+d-1}\right) \subseteq \mathfrak{q}^{n} \subseteq \mathfrak{a}^{n} .
$$

Next let $\mathfrak{a}$ be an arbitrary ideal. Since our assertion holds true for every $\mathfrak{m}$-primary ideal, it follows that

$$
\tau\left(\mathfrak{a}^{n+d-1}\right) \subseteq \bigcap_{t \in \mathbb{N}} \tau\left(\left(\mathfrak{a}+\mathfrak{m}^{t}\right)^{n+d-1}\right) \subseteq \bigcap_{t \in \mathbb{N}}\left(\mathfrak{a}+\mathfrak{m}^{t}\right)^{n}=\mathfrak{a}^{n} .
$$

The second assertion is proved in a similar way.

Tight integral closure vs. a-tight closure. First, we recall the notion of tight integral closure, which was introduced by Hochster.

Definition 2.5 ([Ho2]). Let $R$ be a Noetherian ring, and let $\left\{I_{1}, \ldots, I_{n}\right\}$ be a set of ideals in $R$. An element $x \in R$ is in the tight integral closure $\left\{I_{1}, \ldots, I_{n}\right\}^{*}$ if there exists $c \in R^{\circ}$ such that $c x^{q} \in \sum_{i=1}^{n} I_{i}^{q}$ for all sufficiently large $q=p^{e}$.

In [HWY1], the present authors have studied the F-rationality of Rees algebras $\mathbf{R}(I)=R[I t]$ for $\mathfrak{m}$-primary ideals $I$, jointly with K.-i. Watanabe. One of the main results in [HWY1] is the following theorem, which gives a criterion for F-rationality of Rees algebras in terms of tight integral closure.

Theorem 2.6 (cf. HWY1 Theorem 2.2]). Let (R, m) be an excellent CohenMacaulay normal local ring of characteristic $p>0$ with infinite residue field. Let $I$ be an $\mathfrak{m}$-primary ideal of $R$ and $J$ its minimal reduction. Fix any system of parameters $x_{1}, \ldots, x_{d}$ for $R$ generating $J$ and put $J^{[l]}=\left(x_{1}^{l}, \ldots, x_{d}^{l}\right)$ for $l \geq 1$. Then the Rees algebra $\mathbf{R}(I)=R[I t]$ is F-rational if and only if $\mathbf{R}(I)$ is CohenMacaulay and the following equalities hold:

$$
\left\{I^{d l-r}, x_{1}^{l} R, \ldots, x_{d}^{l} R\right\}^{*}=I^{d l-r}+J^{[l]} \text { for all } l, r \geq 1 \text { with } 1 \leq r \leq d l-1 .
$$

We will show that all tight integral closures appearing in the above theorem can be represented as the form of some "a-tight closure." Namely, we have the following theorem.

Theorem 2.7. Let $(R, \mathfrak{m})$ be an excellent equidimensional reduced local ring of characteristic $p>0$ with $d=\operatorname{dim} R \geq 1$. Also, let $x_{1}, \ldots, x_{d}$ be a system of parameters of $R$, and put $J=\left(x_{1}, \ldots, x_{d}\right) R$. Then we have

$$
\left\{J^{d l-r}, x_{1}^{l} R, \ldots, x_{d}^{l} R\right\}^{*}=\left(x_{1}^{l}, \ldots, x_{d}^{l}\right)^{* J^{r}}
$$

for all integers $l, r \geq 1$.

Before proving the above theorem, we give some corollaries. We now recall that

$$
\overline{I_{1}}+\cdots+\overline{I_{n}} \subseteq\left\{I_{1}, \ldots, I_{n}\right\}^{*} ;
$$

see [Ho1, Proposition 1.4].

Corollary 2.8. Using the same notation as in Theorem 2.7, if $J \subseteq I \subseteq \bar{J}$, then $\left(J^{[l]}\right)^{* J^{r}} \supseteq \overline{J^{d l-r}}+J^{[l]}$ for all $l, r \geq 1$. In particular, we have

(1) $J+\overline{I^{d-1}} \subseteq J^{* I}$. 
(2) If $J^{* I^{r}}=J$, then $I^{d-r} \subseteq J$.

As an application of Theorem 2.7, we can rewrite Theorem 2.6 as follows.

Corollary 2.9. Using the same notation as in Theorem 2.6, the Rees algebra $\mathbf{R}(I)$ is F-rational if and only if $\mathbf{R}(I)$ is Cohen-Macaulay and the following equalities hold:

$$
\left(J^{[l]}\right)^{* I^{r}}=I^{d l-r}+J^{[l]} \text { for all } l, r \geq 1 \text { with } 1 \leq r \leq d l-1 .
$$

Corollary 2.10. Let $R$ be an excellent F-rational local ring with $\operatorname{dim} R=2$. Then for any parameter ideal $J$ of $R$, we have $J^{* J}=\bar{J}$.

Proof. Put $I=\bar{J}$. Then $J$ is a minimal reduction of $I$. Since $\mathbf{R}(I)$ is F-rational by [HWY1. Theorem 3.1], it follows from Theorems 2.6 and 2.7 that $J^{* J}=J^{* I}=$ $I+J=I$, as required.

In the following, we prove Theorem 2.7 , and so we assume that $(R, \mathfrak{m}, k)$ is an excellent equidimensional (not necessarily reduced) local ring of characteristic $p>0$ with $d=\operatorname{dim} R \geq 1$. Also, let $x_{1}, \ldots, x_{d}$ be a system of parameters of $R$ and put $J=\left(x_{1}, \ldots, x_{d}\right) R$. Further, we set $J^{[l]}:=\left(x_{1}^{l}, \ldots, x_{d}^{l}\right)$ for all $l \geq 1$.

Lemma 2.11. Suppose that $x_{1}, \ldots, x_{d}$ form a regular sequence. Then for all integers $l, r \geq 1$ we have

$$
\left(x_{1}^{l}, \ldots, x_{d}^{l}\right):\left(x_{1}, \ldots, x_{d}\right)^{r}=\left(x_{1}, \ldots, x_{d}\right)^{d l-r-d+1}+\left(x_{1}^{l}, \ldots, x_{d}^{l}\right),
$$

that is, $J^{[l]}: J^{r}=J^{d l-r-d+1}+J^{[l]}$.

Proof. The right-hand side is contained in the left-hand side because $J^{d l-r-d+1} J^{r}=$ $J^{d(l-1)+1} \subseteq J^{[l]}$. We must show the opposite inclusion. To do that, let $w=$ $x_{1}^{a_{1}} \cdots x_{d}^{a_{d}}$, where $0 \leq a_{i} \leq l-1$ for all $i$. First suppose that $\sum_{i=1}^{d} a_{i}=d l-r-d$. If we put $b_{i}=l-1-a_{i}$ for all $i$, then $b_{i} \geq 0, \sum_{i=1}^{d} b_{i}=r$ and $w \cdot x_{1}^{b_{1}} \cdots x_{d}^{b_{d}}=$ $x_{1}^{l-1} \cdots x_{d}^{l-1} \notin J^{[l]}$. Next suppose that $\sum_{i=1}^{d} a_{i} \geq d l-r-d+1$. Then for all integers $c_{i} \geq 0$ with $\sum_{i=1}^{d} c_{i}=r$, we have $w \cdot x_{1}^{c_{1}} \cdots x_{d}^{c_{d}}=x_{1}^{a_{1}+c_{1}} \cdots x_{d}^{a_{d}+c_{d}} \in J^{[l]}$, because $\sum_{i=1}^{d}\left(a_{i}+c_{i}\right) \geq d(l-1)+1$. Since $J^{[l]}: J^{r}$ is generated by monomials in $x_{1}, \ldots, x_{d}$, the assertion follows from the above argument.

Using the colon-capturing property of tight closure, we obtain the following.

Corollary 2.12. In the above notation, for all $l, r \geq 1$, we have

$$
J^{[l]}: J^{r} \subseteq\left(J^{d l-r-d+1}+J^{[l]}\right)^{*} .
$$

Proof. First suppose that $R$ is complete and reduced. If we put $S=k\left[\left[x_{1}, \ldots, x_{d}\right]\right]$, then $S$ is a complete regular local domain and $R$ is a finitely generated torsion-free $S$-module. Also, if we put $J_{0}=\left(x_{1}, \ldots, x_{d}\right) S$ and $J_{0}^{[l]}=\left(x_{1}^{l}, \ldots, x_{d}^{l}\right) S$, then $J=J_{0} R$ and $J^{[l]}=J_{0}^{[l]} R$. Using the colon-capturing property of tight closure and the previous lemma, we get

$$
J^{[l]}: J^{r} \subseteq\left(\left(J_{0}^{[l]}: J_{0}^{r}\right) R\right)^{*}=\left(\left(J_{0}^{d l-r-d+1}+J_{0}^{[l]}\right) R\right)^{*}=\left(J^{d l-r-d+1}+J^{[l]}\right)^{*} .
$$

Next we consider the general case. Fix $l, r \geq 1$ and put $K=J^{d l-r-d+1}+J^{[l]}$. Applying the above argument to $\widehat{R}_{\text {red }}=\widehat{R_{\text {red }}}$, we have

$$
J^{[l]} \widehat{R}_{\text {red }}: J^{r} \widehat{R}_{\text {red }} \subseteq\left(K \widehat{R}_{\text {red }}\right)^{*}=(K \widehat{R})^{*} \widehat{R}_{\text {red }},
$$


and hence $J^{[l]} \widehat{R}: J^{r} \widehat{R} \subseteq(K \widehat{R})^{*}$. By [BH, Proposition 10.3.18], we get

$$
J^{[l]}: J^{r}=\left(J^{[l]} \widehat{R}: J^{r} \widehat{R}\right) \cap R=(K \widehat{R})^{*} \cap R=K^{*} \widehat{R} \cap R=K^{*},
$$

as required.

We are now ready to prove Theorem 2.7 .

Proof of Theorem 2.7. Note that $R$ admits a test element $c^{\prime} \in R^{\circ}$, because $R$ is an excellent reduced local ring ([HH2, Theorem 6.1]).

Let $z \in\left(J^{[l]}\right)^{* J^{r}}$. By definition, there exists $c^{\prime \prime} \in R^{\circ}$ such that $c^{\prime \prime} z^{q} J^{r q} \subseteq J^{[l q]}$ for all $q=p^{e}, e \gg 0$. Corollary 2.12 implies that

$$
c^{\prime \prime} z^{q} \in J^{[l q]}: J^{r q} \subseteq\left(J^{(d l-r) q-d+1}+J^{[l q]}\right)^{*},
$$

and hence

$$
c^{\prime} c^{\prime \prime} z^{q} \in J^{(d l-r) q-d+1}+J^{[l q]}
$$

for all $q=p^{e}, e \gg 0$. Take any element $c^{\prime \prime \prime} \in J^{d-1} \cap R^{\circ}$ and put $c=c^{\prime} c^{\prime \prime} c^{\prime \prime \prime} \in R^{\circ}$. Then $c z^{q} \in J^{(d l-r) q}+J^{[l q]}$ for all $q=p^{e}, e \gg 0$. Thus $z \in\left\{J^{d l-r}, x_{1}^{l} R, \ldots, x_{d}^{l} R\right\}^{*}$.

Next we prove the opposite inclusion. Let $w \in\left\{J^{d l-r}, x_{1}^{l} R, \ldots, x_{d}^{l} R\right\}^{*}$. By definition, there exists $c^{\prime \prime} \in R^{\circ}$ such that $c^{\prime \prime} w^{q} \in J^{(d l-r) q}+J^{[l q]}$ for all $q=p^{e}, e \gg 0$. Thus $c^{\prime \prime} w^{q} J^{r q} \subseteq J^{d l q}+J^{[l q]}$.

On the other hand, by virtue of the tight closure Briançon-Skoda theorem [HH1. Theorem 5.4], we have

$$
J^{d l q} \subseteq \overline{J^{d l q}} \subseteq\left(J^{[l q]}\right)^{*} .
$$

Taking a test element $c^{\prime} \in R^{\circ}$, we have $c^{\prime}\left(J^{[l q]}\right)^{*} \subseteq J^{[l q]}$ for all $q=p^{e}, e \gg 0$. In particular, we have $c^{\prime} c^{\prime \prime} w^{q} J^{r q} \subseteq J^{[l q]}$ for all sufficiently large $q=p^{e}$, and thus $w \in\left(J^{[l]}\right)^{* J^{r}}$, as required.

Remark 2.13. Although we can easily see that $J^{* I} R_{\text {red }} \subseteq\left(J R_{\text {red }}\right)^{* I R_{\text {red }}}$ always holds, we do not know whether or not the opposite inclusion holds. If it were true, we could remove the assumption that " $R$ is reduced" in Theorem 2.7.

A characterization of regular local rings. Let $(R, \mathfrak{m}, k)$ be an excellent equidimensional reduced local ring of characteristic $p>0$ with $d=\operatorname{dim} R \geq 1$. Then $R$ is F-rational if and only if $J^{*}=J$ for some (every) parameter ideal $J$ of $R$; see [HH2] and [FW].

Let $J$ be a minimal reduction of $\mathfrak{m}$. Since $J^{*}=J^{* R}$, we have the following increasing sequence of ideals in $R$ :

$$
J \subseteq J^{*} \subseteq J^{* \mathfrak{m}} \subseteq J^{* \mathfrak{m}^{2}} \subseteq \cdots \subseteq J^{* \mathfrak{m}^{d-1}} \subseteq J^{* \mathfrak{m}^{d}}=R,
$$

where the equality on the right follows from the tight closure Briançon-Skoda theorem ([HH1, Theorem 5.4]). So it is natural to ask the following question.

Question 2.14. Let $R$ be an excellent equidimensional local ring of characteristic $p>0$, and let $J$ be a minimal reduction of $\mathfrak{m}$ (in general, a parameter ideal of $R$ ). When does the equality $J^{* \mathfrak{m}^{d-1}}=J$ hold?

In the following, we give a characterization of regular local rings in terms of $\mathfrak{a}$-tight closure, which gives an answer to the above question. See also Section 5 about related problems. 
Theorem 2.15. Let $(R, \mathfrak{m}, k)$ be an excellent equidimensional reduced local ring of characteristic $p>0$ with $d=\operatorname{dim} R \geq 1$, and assume that $k$ is infinite. Then the following conditions are equivalent:

(1) $R$ is regular;

(2) $\tau\left(\mathfrak{m}^{d-1}\right)=R$, i.e., $I^{* \mathfrak{m}^{d-1}}=I$ holds for every ideal $I$ of $R$;

(3) $J^{* \mathfrak{m}^{d-1}}=J$ holds for some parameter ideal $J$ of $R$.

In order to prove Theorem 2.15, we need the following lemma. Note that we do not need to assume that $R$ is excellent in the proof of this lemma.

Lemma 2.16. Let $(R, \mathfrak{m})$ be a regular local ring with $d=\operatorname{dim} R \geq 1$. Then $I^{* \mathfrak{m}^{d-1}}=I$ for every ideal $I$ of $R$.

Proof. Suppose that $I^{* \mathfrak{m}^{d-1}} \neq I$ for some ideal $I$ of $R$. Let $z \in I^{* \mathfrak{m}^{d-1}} \backslash I$. By definition, there exists $c \in R^{\circ}$ such that $c z^{q} \mathfrak{m}^{(d-1) q} \subseteq I^{[q]}$ for all $q=p^{e}, e \gg 0$. Since the Frobenius map $F: R \rightarrow R$ is flat by Kunz' theorem ([Ku] $)$, we have

$$
\mathrm{cm}^{(d-1) q} \subseteq I^{[q]}: z^{q}=(I: z)^{[q]} \subseteq \mathfrak{m}^{[q]},
$$

and hence

$$
c \in \mathfrak{m}^{[q]}: \mathfrak{m}^{(d-1) q}=\mathfrak{m}^{q-d+1}
$$

for all $q=p^{e}, e \gg 0$, by Lemma 2.11. This is a contradiction.

Proof of Theorem 2.15. Note that $R$ is approximately Gorenstein by our assumption. (1) $\Rightarrow(2)$ follows from Lemma 2.16. Also, $(2) \Rightarrow(3)$ is trivial.

Let us prove $(3) \Rightarrow(1)$. Take a parameter ideal $J$ such that $J^{* \mathfrak{m}^{d-1}}=J$. Since $J \subseteq J^{*} \subseteq J^{* m^{d-1}}$, we have $J=J^{*}$. Hence $R$ is F-rational, and thus is Cohen-Macaulay. Then for a minimal reduction $\mathfrak{q}$ of $\mathfrak{m}$, we have $\mathfrak{q}^{* \mathfrak{m}^{d-1}}=\mathfrak{q}$ (see 1.14). Thus we may assume that $J$ is a minimal reduction of $\mathfrak{m}$. Then by virtue of Corollary 2.8, we have $\mathfrak{m} \subseteq J$. This implies that $R$ is regular, as required.

\section{INTERPRETATION OF MULTIPLIER IDEALS VIA $\mathfrak{a}$-TIGHT CLOSURE}

We posed Theorem 2.1 as a prime characteristic analogue of Lipman's "modified Briançon-Skoda theorem" [Li]. The original form of "modified Briançon-Skoda" is stated in terms of what is called "adjoint ideals" by Lipman. Recently, this notion is reformulated in the theory of "multiplier ideals" from a different point of view and plays an important role in birational algebraic geometry; see [Ei], LLa]. Actually, one can define multiplier ideals with "rational coefficients"; cf. Section 6.

Definition 3.1. Let $Y$ be a normal $\mathbb{Q}$-Gorenstein variety over a field of characteristic zero and let $\mathfrak{a} \subset \mathcal{O}_{Y}$ be a nonzero ideal sheaf. Let $f: X \rightarrow Y$ be a log resolution of the ideal $\mathfrak{a}$, that is, a resolution of singularities of $Y$ such that the ideal sheaf $\mathfrak{a} \mathcal{O}_{X}$ is invertible, say, $\mathfrak{a} \mathcal{O}_{X}=\mathcal{O}_{X}(-Z)$ for an effective divisor $Z$ on $X$, and that the union $\operatorname{Exc}(f) \cup \operatorname{Supp}(Z)$ of the $f$-exceptional locus and the support of $Z$ is a simple normal crossing divisor. Given a rational number $t \geq 0$, the multiplier ideal $\mathcal{J}(t \cdot \mathfrak{a})=\mathcal{J}\left(\mathfrak{a}^{t}\right)$ associated to $t$ and $\mathfrak{a}$ is defined to be the ideal sheaf

$$
\mathcal{J}(t \cdot \mathfrak{a})=f_{*} \mathcal{O}_{X}\left(\left\lceil K_{X / Y}-t Z\right\rceil\right)
$$

in $\mathcal{O}_{Y}$, where the $\mathbb{Q}$-divisor $K_{X / Y}=K_{X}-f^{*} K_{Y}$ is the discrepancy of $f$. For $t=1$, we just denote $\mathcal{J}(\mathfrak{a}):=\mathcal{J}(1 \cdot \mathfrak{a})$. This definition is independent of the choice of a $\log$ resolution $f: X \rightarrow Y$ of $\mathfrak{a}$. 
Remark 3.2. (1) If $\mathfrak{b}=\mathfrak{a}^{n}$ for a nonnegative integer $n$, then $\mathcal{J}(t \cdot \mathfrak{b})=\mathcal{J}(t n \cdot \mathfrak{a})$, and this justifies the notation $\mathcal{J}\left(\mathfrak{a}^{t}\right)$ with "formal exponent" $t$. Henceforth we prefer the exponential notation $\mathcal{J}\left(\mathfrak{a}^{t}\right)$ rather than $\mathcal{J}(t \cdot \mathfrak{a})$; cf. Section 6 .

(2) Multiplier ideals $\mathcal{J}(\mathfrak{a})$ have properties similar to those of the ideals $\tau(\mathfrak{a})$; see Proposition 1.11. Namely, if $\mathfrak{b} \subseteq \mathfrak{a}$, then $\mathcal{J}(\mathfrak{b}) \subseteq \mathcal{J}(\mathfrak{a})$, and if $\mathfrak{b}$ is a reduction of $\mathfrak{a}$, then the equality $\mathcal{J}(\mathfrak{b})=\mathcal{J}(\mathfrak{a})$ holds; $\mathcal{J}(\mathfrak{a}) \mathfrak{b} \subseteq \mathcal{J}(\mathfrak{a} \mathfrak{b})$ for any $\mathfrak{a}, \mathfrak{b}$, and if $\mathfrak{b}$ is locally principal, then the equality $\mathcal{J}(\mathfrak{a}) \mathfrak{b}=\mathcal{J}(\mathfrak{a} \mathfrak{b})$ holds; $\mathcal{J}(\mathfrak{a}) \neq 0$ as long as $\mathfrak{a} \neq 0$; and if $Y$ has only $\log$ terminal singularities, then $\mathfrak{a} \subseteq \mathcal{J}(\mathfrak{a})$. Also, using vanishing theorems in characteristic zero, one can prove a "modified BriançonSkoda theorem" ([Li, Theorem 1.4.1], [La]): If $\mathfrak{a}$ is generated by $r$ elements, then $\mathcal{J}\left(\mathfrak{a}^{n+r-1}\right) \subseteq \mathfrak{a}^{n}$ for every $n \geq 0$; cf. Theorem 2.1. Later in Section 4, we study more about similarity of the ideals $\tau(\mathfrak{a})$ and $\mathcal{J}(\mathfrak{a})$.

3.3. Reduction to prime characteristic. It is proved in [Ha2] and [Sm2] that the multiplier ideal $\mathcal{J}(R)$ of the unit ideal in a normal $\mathbb{Q}$-Gorenstein ring $R$ essentially of finite type over a field of characteristic zero coincides, after reduction to characteristic $p \gg 0$, with the test ideal $\tau(R)$. We generalize this result in Theorem 3.4 below. To state the result, we have to begin with a ring $R$ and an ideal $\mathfrak{a}$ in characteristic zero, and reduce them to characteristic $p \gg 0$ together with a log resolution $f: X \rightarrow \operatorname{Spec} R$ of $\mathfrak{a}$.

Let $R$ be an algebra essentially of finite type over a field $k$ of characteristic zero, and let $\mathfrak{a} \subseteq R$ be an ideal. One can choose a finitely generated $\mathbb{Z}$-algebra $A$ contained in $k$ and a subalgebra $R_{A}$ of $R$ essentially of finite type over $A$ such that the natural map $R_{A} \otimes_{A} k \rightarrow R$ is an isomorphism and $\mathfrak{a}_{A}=\mathfrak{a} \cap R_{A}$ generates the ideal $\mathfrak{a}$ of $R$. For a maximal ideal $\mu$ of $A$, we consider the base change to its residue field $\kappa=\kappa(\mu)$ over $A$ to get a prime characteristic ring $R_{\kappa}=R_{A} \otimes_{A} \kappa$ and an ideal $\mathfrak{a}_{\kappa}=\mathfrak{a}_{A} R_{\kappa}$. The data consisting of $\kappa=\kappa(\mu), R_{\kappa}, \mathfrak{a}_{\kappa}$ is considered to be a "prime characteristic model" of the original data in characteristic zero, and we refer to such $\left(\kappa, R_{\kappa}, \mathfrak{a}_{\kappa}\right)$ for maximal ideals $\mu$ in a suitable dense open subset of $\operatorname{Spec} A$ as "reduction to characteristic $p \gg 0$ " of $(k, R, \mathfrak{a})$. Furthermore, given a morphism of schemes essentially of finite type over $k$ (and even more, a commutative diagram consisting of a finite collection thereof), e.g., a $\log$ resolution $f: X \rightarrow \operatorname{Spec} R$ of $\mathfrak{a}$, we can reduce this entire setup to characteristic $p \gg 0$. (See [Ha1], Ha2], HH3, Sm1, Sm2 for more details.) We use the phrase "in characteristic $p \gg 0$ " when we speak of such a setup reduced from characteristic zero to characteristic $p \gg 0$.

The main result of this section is the following theorem, which ensures the correspondence of the ideal $\tau(\mathfrak{a})$ and the multiplier ideal $\mathcal{J}(\mathfrak{a})$. See Theorem 6.7 for a generalization of this theorem to the case of "rational coefficients".

Theorem 3.4. Let $R$ be a normal $\mathbb{Q}$-Gorenstein local ring essentially of finite type over a field, and let $\mathfrak{a}$ be a nonzero ideal. Assume that $\mathfrak{a} \subseteq R$ is reduced from characteristic zero to characteristic $p \gg 0$, together with a log resolution of singularities $f: X \rightarrow Y=\operatorname{Spec} R$ such that $\mathfrak{a} \mathcal{O}_{X}=\mathcal{O}_{X}(-Z)$ is invertible. Then

$$
\tau(\mathfrak{a})=H^{0}\left(X, \mathcal{O}_{X}\left(\left\lceil K_{X / Y}\right\rceil-Z\right)\right) .
$$

3.5. The remainder of this section is devoted to proving Theorem 3.4. Our strategy is to reduce to the case where the ring $R$ is quasi-Gorenstein by passing to a canonical covering; see $[\mathrm{T}]$ for a direct proof which does not use a canonical covering. Let $(R, \mathfrak{m})$ be a normal $\mathbb{Q}$-Gorenstein local ring with a canonical module $\omega_{R}$, and let $r$ be the least positive integer such that the $r$ th symbolic power $\omega_{R}^{(r)}$ of $\omega_{R}$ is 
isomorphic to $R$. Given a fixed isomorphism $\omega_{R}^{(r)} \cong R$, one has a natural ring structure of $S=\bigoplus_{i=0}^{r-1} \omega_{R}^{(i)}$. This is a quasi-Gorenstein local ring (i.e., $\omega_{S} \cong S$ ) with the maximal ideal $\mathfrak{m} \oplus \bigoplus_{i=1}^{r-1} \omega_{R}^{(i)}$, and we call $S$ a canonical covering of $R$.

The following two lemmas make it possible to reduce the proof of the theorem to the quasi-Gorenstein case.

Lemma 3.6. Let $(R, \mathfrak{m})$ be a normal local ring of characteristic $p>0, S$ a canonical covering of $R$ as above, and assume that $r$ is not divisible by $p$. Then for any ideal $\mathfrak{a} \subseteq R$,

$$
\tau(\mathfrak{a} S) \cap R=\tau(\mathfrak{a}) .
$$

Proof. Identical to the case where $\mathfrak{a}=R$; see [Ha2, Section 2], and also [Sm2].

The following lemma is also proved entirely as in the same way as the case where $\mathfrak{a}=R$ [Sm2 Proposition 3.2], but we include the proof for the sake of completeness.

Lemma 3.7 (cf. [Sm1]). Let $(R, \mathfrak{m})$ be a normal local ring essentially of finite type over a field of characteristic zero, and let $S$ be a canonical covering of $R$ as above. Then, for any ideal $\mathfrak{a} \subseteq R$ and any rational number $t \geq 0$,

$$
\mathcal{J}\left((\mathfrak{a} S)^{t}\right) \cap R=\mathcal{J}\left(\mathfrak{a}^{t}\right) .
$$

Proof. Let $\pi:$ Spec $S \rightarrow \operatorname{Spec} R$ be the canonical covering, and let $f: X \rightarrow \operatorname{Spec} R$ and $g: Y \rightarrow \operatorname{Spec} S$ be $\log$ resolutions of $\mathfrak{a}$ and $\mathfrak{a} S$, respectively, which make the following diagram commute:

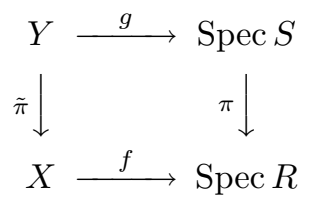

Let $E$ be the reduced divisor on $X$ supported on $\operatorname{Exc}(f) \cup \operatorname{Supp}(Z)$ and let $G$ be the reduced divisor with the same support as $\tilde{\pi}^{*} E$. Then the $\log$ ramification formula (see, e.g., $\mathrm{Ka}$ ) tells us that

$$
K_{Y / S}+G=\tilde{\pi}^{*}\left(K_{X / R}+E\right)+P
$$

for some effective divisor $P$ on $Y$ such that $\operatorname{codim}(\tilde{\pi}(P), X) \geq 2$.

Now let $u \in \mathcal{J}\left(\mathfrak{a}^{t}\right)=H^{0}\left(X, \mathcal{O}_{X}\left(\left\lceil K_{X / R}-t Z\right\rceil\right)\right)$, i.e., $\operatorname{div}_{X}(u)+\left\lceil K_{X / R}-t Z\right\rceil \geq 0$. Since $\operatorname{Supp}\left(K_{X / R}-t Z\right) \subseteq E$, one has $K_{X / R}-t Z+E \geq\left\lceil K_{X / R}-t Z\right\rceil$, so that

$$
\operatorname{div}_{X}(u)+K_{X / R}+E-t Z \geq 0
$$

and this is a strict inequality for the coefficient in each irreducible component of $E$. Pulling this back by $\tilde{\pi}$ and applying (3.7.1) give an inequality

$$
\operatorname{div}_{Y}(u)+K_{Y / S}+G-t \tilde{\pi}^{*} Z \geq 0
$$

which is a strict inequality for the coefficient in each irreducible component of $G$. Since $G$ is reduced, it follows that $\operatorname{div}_{Y}(u)+\left\lceil K_{Y / S}-t \tilde{\pi}^{*} Z\right\rceil \geq 0$. Hence $u \in H^{0}\left(X, \mathcal{O}_{Y}\left(\left\lceil K_{Y / S}-t \tilde{\pi}^{*} Z\right\rceil\right)\right)=\mathcal{J}\left((\mathfrak{a} S)^{t}\right)$.

Conversely, let $u \in \mathcal{J}\left((\mathfrak{a} S)^{t}\right) \cap R$ and fix any prime divisor $D$ on $X$. To prove $u \in \mathcal{J}\left(\mathfrak{a}^{t}\right)$, it is enough to show that $a:=v_{D}(u)+\operatorname{coeff}_{D}\left(\left\lceil K_{X / R}-t Z\right\rceil\right)$ is nonnegative. If $D$ is not $f$-exceptional, this follows because $\tilde{\pi}$ is étale and finite at the generic point of $D$ and $\operatorname{div}_{Y}(u)+\left\lceil K_{Y / S}-t \tilde{\pi}^{*} Z\right\rceil \geq 0$ by $u \in \mathcal{J}\left((\mathfrak{a} S)^{t}\right)$. Now let 
$D$ be $f$-exceptional, $F$ any prime divisor on $Y$ dominating $D$ (this in particular implies that $F \nsubseteq \operatorname{Supp}(P))$, and let $e:=\operatorname{coeff}_{F}\left(\tilde{\pi}^{*} D\right)$. Then

$$
v_{F}(u)+\operatorname{coeff}_{F}\left(\tilde{\pi}^{*}\left(\left\lceil K_{X / R}-t Z\right\rceil\right)\right)=e a .
$$

On the other hand, since $\operatorname{div}_{Y}(u)+\left\lceil K_{Y / S}-t \tilde{\pi}^{*} Z\right\rceil \geq 0$, it follows from (3.7.1) that $\operatorname{div}_{Y}(u)+\left\lceil\tilde{\pi}^{*}\left(K_{X / R}+E\right)-G+P-t \tilde{\pi}^{*} Z\right\rceil \geq 0$. Since $F \nsubseteq \operatorname{Supp}(P)$, this implies that

$$
v_{F}(u)+\operatorname{coeff}_{F}\left(\left\lceil\tilde{\pi}^{*}\left(K_{X / R}-t Z\right)\right\rceil\right) \geq \operatorname{coeff}_{F}\left(-\tilde{\pi}^{*} E+G\right)=-e+1 .
$$

It follows from (3.7.2) and (3.7.3) that $e a \geq-e+1$, so that $a \geq 0$, as required.

Remark. The proof of Lemma 3.7 works not only for canonical coverings but also under a weaker assumption that $R \hookrightarrow S$ is finite and étale in codimension 1 . It is desirable that Lemma 3.6 also holds for finite extensions that are étale in codimension 1 , and this issue has been recently settled by Takagi $([\mathrm{HT}],[\mathrm{T}])$.

Proposition 3.8. Let $(R, \mathfrak{m})$ be a d-dimensional normal local ring of characteristic $p>0$, and let $\mathfrak{a}$ be a nonzero ideal. Let $f: X \rightarrow \operatorname{Spec} R$ be a proper birational morphism from a normal scheme $X$ such that $\mathfrak{a} \mathcal{O}_{X}=\mathcal{O}_{X}(-Z)$ is an invertible sheaf, and denote the closed fiber of $f$ by $E=f^{-1}(\mathfrak{m})$. Then one has an inclusion

$$
\operatorname{Ker}\left(H_{\mathfrak{m}}^{d}(R) \stackrel{\delta}{\longrightarrow} H_{E}^{d}\left(\mathcal{O}_{X}(Z)\right)\right) \subseteq 0_{H_{\mathfrak{m}}^{d}(R)}^{* \mathfrak{a}},
$$

where $\delta: H_{\mathfrak{m}}^{d}(R) \rightarrow H_{E}^{d}\left(\mathcal{O}_{X}(Z)\right)$ is an edge map $H_{\mathfrak{m}}^{d}(R) \rightarrow H_{E}^{d}\left(\mathcal{O}_{X}\right)$ of the spectral sequence $H_{\mathfrak{m}}^{i}\left(R^{j} f_{*} \mathcal{O}_{X}\right) \Longrightarrow H_{E}^{i+j}\left(\mathcal{O}_{X}\right)$ followed by the natural map $H_{E}^{d}\left(\mathcal{O}_{X}\right) \rightarrow$ $H_{E}^{d}\left(\mathcal{O}_{X}(Z)\right)$.

Proof. First note that, for any $q=p^{e}$ and any $c \in \mathfrak{a}^{q} \subseteq H^{0}\left(X, \mathcal{O}_{X}(-q Z)\right)$, we have the following commutative diagram with exact rows:

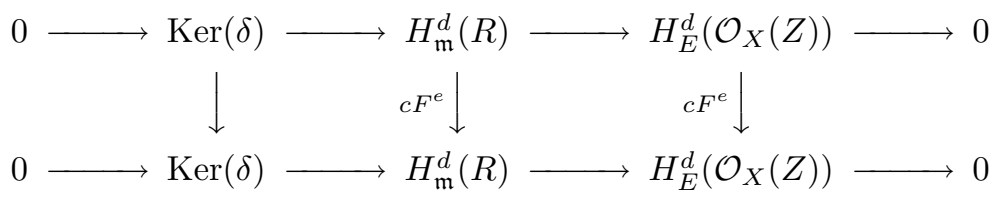

Then $\mathfrak{a}^{q} F^{e}(\operatorname{Ker}(\delta)) \subseteq \operatorname{Ker}(\delta)$ for all $q=p^{e}$, and the conclusion follows from Proposition 1.15 .

By virtue of Lemmas 3.6 and 3.7, it is sufficient to prove Theorem 3.4 in the case where $R$ is quasi-Gorenstein, i.e., $\omega_{R} \cong R$. In this case, however, the assertion of Theorem 3.4 coincides with the following.

Theorem 3.9. Let $(R, \mathfrak{m})$ be a d-dimensional normal local ring essentially of finite type over a field of characteristic $p$, and let $\mathfrak{a}$ be a nonzero ideal. Assume that $\mathfrak{a} \subseteq R$ is reduced from characteristic zero to characteristic $p \gg 0$, together with a resolution of singularities $f: X \rightarrow Y=\operatorname{Spec} R$ such that $\mathfrak{a} \mathcal{O}_{X}=\mathcal{O}_{X}(-Z)$ is invertible. Then

$$
0_{H_{\mathfrak{m}}^{d}(R)}^{* \mathfrak{a}}=\operatorname{Ker}\left(H_{\mathfrak{m}}^{d}(R) \stackrel{\delta}{\longrightarrow} H_{E}^{d}\left(\mathcal{O}_{X}(Z)\right)\right),
$$

where $E$ is the closed fiber of $f$ and $\delta$ is the edge map as in 3.8, or dually,

$$
\operatorname{Ann}_{\omega_{R}}\left(0_{H_{\mathfrak{m}}^{d}(R)}^{* \mathfrak{a}}\right)=H^{0}\left(X, \omega_{X}(-Z)\right) \text { in } \omega_{R} .
$$


Proof. First, let us discuss the situation in characteristic zero before reduction to characteristic $p \gg 0$. In characteristic zero, we choose a nonzero element $c \in \mathfrak{a}$ such that $R_{c}$ is regular, and a $\log$ resolution $f: X \rightarrow \operatorname{Spec} R$ of the ideal $c \mathfrak{a}$. Then $\mathfrak{a} \mathcal{O}_{X}=\mathcal{O}_{X}(-Z)$ for an effective divisor $Z$ on $X$, and $\operatorname{Supp}\left(Z+\operatorname{div}_{X}(c)\right)$ is a simple normal crossing divisor. We choose an $f$-ample $f$-exceptional $\mathbb{Q}$-Cartier divisor $D$ and a sufficiently small rational number $\varepsilon>0$ such that $\left\lfloor\tilde{Z}+\varepsilon \operatorname{div}_{X}(c)\right\rfloor=Z$, where $\tilde{Z}=Z-D$.

Now we reduce the entire setup as above to characteristic $p \gg 0$, and switch the notation to denote things after reduction modulo $p$. Let the ideal $\mathfrak{a}$ be generated by $r$ elements. Since $R_{c}$ is regular, some power $c^{s}$ of $c(\in \mathfrak{a})$ is a usual test element and also an $\mathfrak{a}$-test element. Also, since $-\tilde{Z}$ is $f$-ample, $\mathcal{K}=\bigoplus_{n>0} H^{0}\left(X, \omega_{X}(-\lfloor n \tilde{Z}\rfloor)\right)$ is a finitely generated module over $\mathcal{R}=\bigoplus_{n \geq 0} H^{0}\left(X, \mathcal{O}_{X}(-n \tilde{Z})\right)$. Say $\mathcal{K}$ is generated in degree $\leq n_{0}$.

Since we are working in characteristic $p \gg 0$, the $e$-times iterated Frobenius map

$$
F^{e}: H_{E}^{d}\left(\mathcal{O}_{X}(\tilde{Z})\right) \rightarrow H_{E}^{d}\left(\mathcal{O}_{X}\left(p^{e}\left(\tilde{Z}+\varepsilon \operatorname{div}_{X}(c)\right)\right)\right.
$$

is injective for all $q=p^{e}$ by Proposition 3.6 and Corollary 3.8 of [Ha1]; see also [Ha2, Discussion 4.6] and [MS]. This implies that the map

$$
c^{m} F^{e}: H_{E}^{d}\left(\mathcal{O}_{X}(Z)\right)=H_{E}^{d}\left(\mathcal{O}_{X}(\tilde{Z})\right) \rightarrow H_{E}^{d}\left(\mathcal{O}_{X}\left(p^{e} \tilde{Z}\right)\right)
$$

is injective for all sufficiently large $e \in \mathbb{N}$ such that $p^{e} \varepsilon \geq m:=r+2 s+n_{0}-1$. For such $q=p^{e} \gg 0$, we consider the following commutative diagram with exact rows:

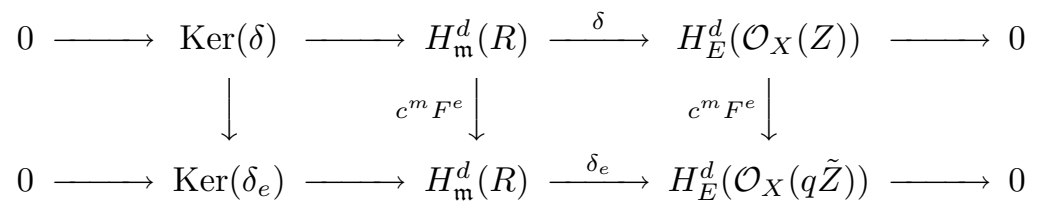

Here, $\operatorname{Ker}\left(\delta_{e}\right)$ is considered to be the annihilator in $H_{\mathfrak{m}}^{d}(R)$ of $H^{0}\left(X, \omega_{X}(-\lfloor q \tilde{Z}\rfloor)\right)$ viewed as a submodule of $\omega_{R}$, with respect to the duality pairing $\omega_{R} \times H_{\mathfrak{m}}^{d}(R) \rightarrow$ $H_{\mathfrak{m}}^{d}\left(\omega_{R}\right) \cong E_{R}(R / \mathfrak{m})$.

Now, if $\xi \in H_{\mathfrak{m}}^{d}(R)$ is not in $\operatorname{Ker}(\delta)$, then

$$
c^{m} \xi^{q} \notin \operatorname{Ker}\left(\delta_{e}\right)=\operatorname{Ann}_{H_{\mathfrak{m}}^{d}(R)} H^{0}\left(X, \omega_{X}(-\lfloor q \tilde{Z}\rfloor)\right)
$$

for all $q=p^{e} \gg 0$ by the above commutative diagram. Then for all sufficiently large $q=p^{e}\left(\geq n_{0}\right)$, there exists an integer $n$ with $0 \leq n \leq n_{0}$ such that

$$
c^{m} \xi^{q} \notin\left[0: H^{0}\left(X, \mathcal{O}_{X}((n-q) \tilde{Z})\right)\right]_{H_{\mathfrak{m}}^{d}(R)},
$$

since $\mathcal{K}$ is generated in degree $\leq n_{0}$ as a graded $\mathcal{R}$-module. Hence it follows from $H^{0}\left(X, \mathcal{O}_{X}((n-q) \tilde{Z})\right) \subseteq H^{0}\left(X, \mathcal{O}_{X}((n-q) Z)\right) \subseteq \overline{\mathfrak{a}^{q-n_{0}}}$ that $c^{m} \xi^{q} \overline{\mathfrak{a}^{q-n_{0}}} \neq 0$. On the other hand, since $c \in \mathfrak{a}$ and $c^{s} \in \tau(R)$,

$$
c^{m-s} \overline{\mathfrak{a}^{q-n_{0}}}=c^{r+s+n_{0}-1} \overline{\mathfrak{a}^{q-n_{0}}} \subseteq \tau(R) \overline{\mathfrak{a}^{q+r-1}} \subseteq \mathfrak{a}^{q}
$$

by the tight closure Briançon-Skoda theorem. Thus we have that $c^{s} \xi^{q} \mathfrak{a}^{q} \neq 0$. But this implies that $\xi \notin 0_{H_{\mathfrak{m}}^{d}(R)}^{* \mathfrak{a}}$, since $c^{s}$ is an $\mathfrak{a}$-test element (cf. Theorem 1.13).

Consequently, we have $0_{H_{\mathrm{m}}^{d}(R)}^{* \mathfrak{a}} \subseteq \operatorname{Ker}(\delta)$. The reverse inclusion follows from Proposition 3.8, and we are done. 


\section{Properties of the ideal $\tau(\mathfrak{a})$ Analogous to multiplier ideals}

In this section we prove some properties of the ideal $\tau(\mathfrak{a})$ analogous to those of the multiplier ideal $\mathcal{J}(\mathfrak{a})$, which are found in DEL, How, and in Lazarsfeld's lecture notes [La; see also similar results for "tight closure for pairs" in [T]. The results in this section are proved in any fixed characteristic $p>0$. However, in view of Theorem 3.4, we can also say that they provide characteristic $p$ proofs of the properties of multiplier ideals in characteristic zero.

We also want to remark that the results in this section can be generalized to "rational coefficients" (see Section 6), just like those for multiplier ideals.

Theorem 4.1 (Restriction theorem, cf. La]). Let $(R, \mathfrak{m})$ be a normal $\mathbb{Q}$-Gorenstein complete local ring of characteristic $p>0$ and let $x \in \mathfrak{m}$ be a non-zero-divisor of $R$. Let $S=R / x R$ and assume that $S$ is normal. Then for any ideal $\mathfrak{a}$ of $R$,

$$
\tau(\mathfrak{a} S) \subseteq \tau(\mathfrak{a}) S
$$

Proof. Let $E_{R}=E_{R}(R / \mathfrak{m})$ and $E_{S}=E_{S}(S / \mathfrak{m} S)$ be the injective envelopes of residue fields of $R$ and $S$, respectively. Then one has $E_{S} \cong(0: x)_{E_{R}} \subset E_{R}$. We first prove the following claim, viewing $E_{S}$ as a submodule of $E_{R}$ via this inclusion.

Claim 4.1.1. $0_{E_{R}}^{* \mathfrak{a}} \cap E_{S} \subseteq 0_{E_{S}}^{* \mathfrak{a} S}$.

Proof of Claim 4.1.1. Since $R$ is normal, we can choose an a-test element $c$ whose image $\bar{c}$ in $S$ is nonzero by Proposition 1.7. Then we have the following commutative diagram for each $q=p^{e}$ (see the proof of [HW] Theorem 4.9]):

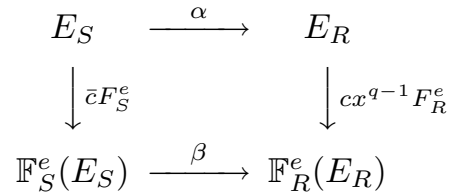

The map $\alpha: E_{S} \rightarrow E_{R}$ is the inclusion map mentioned above. Note also that $\mathbb{F}_{R}^{e}\left(E_{R}\right) \cong H_{\mathfrak{m}}^{d}\left(\omega_{R}^{(q)}\right)$ and $\mathbb{F}_{S}^{e}\left(E_{S}\right) \cong H_{\mathfrak{m}}^{d-1}\left(\omega_{S}^{(q)}\right)$ by [Wa], and $\beta: \mathbb{F}_{S}^{e}\left(E_{S}\right) \rightarrow \mathbb{F}_{R}^{e}\left(E_{R}\right)$ arises as a connecting homomorphism of the long exact sequence

$$
\cdots \rightarrow H_{\mathfrak{m}}^{d-1}\left(\omega_{R}^{(q)}\right) \stackrel{x}{\rightarrow} H_{\mathfrak{m}}^{d-1}\left(\omega_{R}^{(q)}\right) \rightarrow H_{\mathfrak{m}}^{d-1}\left(\omega_{S}^{(q)}\right) \stackrel{\beta}{\rightarrow} H_{\mathfrak{m}}^{d}\left(\omega_{R}^{(q)}\right) \stackrel{x}{\rightarrow} H_{\mathfrak{m}}^{d}\left(\omega_{R}^{(q)}\right) \rightarrow 0
$$

associated to $0 \rightarrow \omega_{R}^{(q)} \stackrel{x}{\rightarrow} \omega_{R}^{(q)} \rightarrow \omega_{R}^{(q)} / x \omega_{R}^{(q)} \rightarrow 0$ ([W] $)$. It then follows that $\operatorname{Ker}(\beta) \cong H_{\mathfrak{m}}^{d-1}\left(\omega_{R}^{(q)}\right) / x H_{\mathfrak{m}}^{d-1}\left(\omega_{R}^{(q)}\right)$ is a proper $S$-submodule of $H_{\mathfrak{m}}^{d-1}\left(\omega_{S}^{(q)}\right)$. Hence we can show as in the proof of Proposition 1.15 that $\operatorname{Ker}(\beta)$ is annihilated by an element $\bar{d} \in S^{\circ}$.

Now let $\xi \in 0_{E_{R}}^{* \mathfrak{a}} \cap E_{S}$. Then $c F_{R}^{e}(\xi) \mathfrak{a}^{q}=0$ in $\mathbb{F}_{R}^{e}\left(E_{R}\right)$ for all $q=p^{e}$, since $c$ is an $\mathfrak{a}$-test element. This implies that $\bar{c} F_{S}^{e}(\xi) \mathfrak{a}^{q} \subseteq \operatorname{Ker}(\beta)$ by the above commutative diagram. Therefore $\bar{c} \bar{d} F_{S}^{e}(\xi)(\mathfrak{a} S)^{q}=0$ for all $q=p^{e}$ with $\bar{c} \bar{d} \in S^{\circ}$, whence $\xi \in 0_{E_{S}}^{* \mathfrak{a} S}$, as claimed.

We continue the proof of Theorem 4.1. Since $R$ is complete, $0_{E_{R}}^{* \mathfrak{a}}=(0: \tau(\mathfrak{a}))_{E_{R}}$, so that

$$
0_{E_{R}}^{* \mathfrak{a}} \cap E_{S}=(0: \tau(\mathfrak{a})+x R)_{E_{R}}=\left(0: \frac{\tau(\mathfrak{a})+x R}{x R}\right)_{E_{S}}=(0: \tau(\mathfrak{a}) S)_{E_{S}} .
$$

Hence by Claim 4.1.1, we conclude that $\tau(\mathfrak{a} S) \subseteq \operatorname{Ann}_{S}\left(0_{E_{R}}^{* \mathfrak{a}} \cap E_{S}\right)=\tau(\mathfrak{a}) S$. 
Remark 4.2. Theorem 4.1 implies that F-regularity "deforms" in $\mathbb{Q}$-Gorenstein rings [AKM]. Namely, if $R$ is $\mathbb{Q}$-Gorenstein, $x \in \mathfrak{m}$ is a non-zero-divisor and $S=$ $R / x R$ is F-regular, then $R$ is also F-regular. This result fails in the absence of $\mathbb{Q}$-Gorensteinness [Si]. On the other hand, the F-regularity of $R$ does not imply the F-regularity of $S=R / x R$, and this suggests that the containment $\tau(\mathfrak{a} S) \subseteq \tau(\mathfrak{a}) S$ is far from an equality in general; see also [HW] Theorem 4.9].

Our next objective is to apply Theorem 4.1 to show the property of $\tau(\mathfrak{a})$ called "subadditivity" (Theorem 4.5), which was established for multiplier ideals in regular rings by Demailly, Ein and Lazarsfeld [DEL]. Our strategy is to mimic the idea of "restriction to the diagonal" used in [DEL]. We first prove the following fact.

Lemma 4.3. Let $R=k\left[\left[x_{1}, \ldots, x_{r}\right]\right]$ and $S=k\left[\left[y_{1}, \ldots, y_{s}\right]\right]$ be complete regular local rings with residue field $k$, and let $T=R \hat{\otimes}_{k} S=k\left[\left[x_{1}, \ldots, x_{r}, y_{1}, \ldots, y_{s}\right]\right]$ be their complete tensor product. Then for any ideals $\mathfrak{a} \subset R$ and $\mathfrak{b} \subset S$, we have that $(\mathfrak{a} \otimes S) \cap(R \otimes \mathfrak{b})=(\mathfrak{a} \otimes \mathfrak{b}) T$ in $T$.

Proof. We regard $R$ and $S$ as subrings of $T$ via the natural ring homomorphisms $R \hookrightarrow T$ and $S \hookrightarrow T$. Then what is to be proved is that $\mathfrak{a} T \cap \mathfrak{b} T=\mathfrak{a} \mathfrak{b} T$. To prove this we may assume, without loss of generality, that $\mathfrak{b}$ is a proper ideal of $S$.

First we note that the composition $R \rightarrow T \rightarrow T / \mathfrak{b} T$ of the natural maps is a flat ring extension. Indeed, $R=R \otimes_{k} k \rightarrow R \otimes_{k} S / \mathfrak{b}$ is flat, since $k \rightarrow S / \mathfrak{b}$ is flat by $\mathfrak{b} \cap k=0$, and the completion map $R \otimes_{k} S / \mathfrak{b} \rightarrow T / \mathfrak{b} T$ is also flat.

Now let $F_{\bullet}$ be an $R$-free resolution of $R / \mathfrak{a}$. Then $F_{\bullet} \otimes_{R} T$ is a $T$-free resolution of $T / \mathfrak{a} T$, since $T$ is flat over $R$. Since $T / \mathfrak{b} T$ is also flat over $R$, one has that $\operatorname{Tor}_{i}^{T}(T / \mathfrak{a} T, T / \mathfrak{b} T)=H_{i}\left(\left(F_{\bullet} \otimes_{R} T\right) \otimes_{T} T / \mathfrak{b}\right)=H_{i}\left(F_{\bullet} \otimes_{R} T / \mathfrak{b} T\right)=0$ for $i>0$. In particular, $(\mathfrak{a} T \cap \mathfrak{b} T) / \mathfrak{a} \mathfrak{b} T \cong \operatorname{Tor}_{1}^{T}(T / \mathfrak{a} T, T / \mathfrak{b} T)=0$. Thus we conclude that $\mathfrak{a} T \cap \mathfrak{b} T=\mathfrak{a} \mathfrak{b} T$, as required.

Proposition 4.4. Let $k$ be a field of characteristic $p$, and let $R=k\left[\left[x_{1}, \ldots, x_{r}\right]\right]$, $S=k\left[\left[y_{1}, \ldots, y_{s}\right]\right]$ and $T=R \hat{\otimes}_{k} S=k\left[\left[x_{1}, \ldots, x_{r}, y_{1}, \ldots, y_{s}\right]\right]$ be as in Lemma 4.3. Then, for any ideals $\mathfrak{a} \subset R$ and $\mathfrak{b} \subset S$,

$$
\tau((\mathfrak{a} \otimes \mathfrak{b}) T) \subseteq(\tau(\mathfrak{a}) \otimes \tau(\mathfrak{b})) T .
$$

Proof. Let us denote the injective envelopes of the residue fields of $R, S, T$ by $E_{R}, E_{S}, E_{T}$, respectively. Then we can describe them in terms of inverse polynomials as $E_{R}=\left(x_{1} \cdots x_{r}\right)^{-1} k\left[x_{1}^{-1}, \ldots, x_{r}^{-1}\right], E_{S}=\left(y_{1} \cdots y_{s}\right)^{-1} k\left[y_{1}^{-1}, \ldots, y_{s}^{-1}\right]$, $E_{T}=\left(x_{1} \cdots x_{r} y_{1} \cdots y_{s}\right)^{-1} k\left[x_{1}^{-1}, \ldots, x_{r}^{-1}, y_{1}^{-1}, \ldots, y_{s}^{-1}\right]$; so, in particular, $E_{T}=$ $E_{R} \otimes_{k} E_{S}$. Then it is easy to see that $0_{E_{R}}^{* \mathfrak{a}} \otimes E_{S}+E_{R} \otimes 0_{E_{S}}^{* \mathfrak{b}} \subseteq 0_{E_{T}}^{*(\mathfrak{a} \otimes \mathfrak{b}) T}$. Hence

$$
\begin{aligned}
\tau((\mathfrak{a} \otimes \mathfrak{b}) T) & \subseteq \operatorname{Ann}_{T}\left(0_{E_{R}}^{* \mathfrak{a}} \otimes E_{S}\right) \cap \operatorname{Ann}_{T}\left(E_{R} \otimes 0_{E_{S}}^{* \mathfrak{b}}\right) \\
& =(\tau(\mathfrak{a}) \otimes S) \cap(R \otimes \tau(\mathfrak{b}))=(\tau(\mathfrak{a}) \otimes \tau(\mathfrak{b})) T
\end{aligned}
$$

by Lemma 4.3 .

Theorem 4.5 (Subadditivity, cf. [DEL]). Let $(R, \mathfrak{m})$ be a complete regular local ring of characteristic $p>0$. Then for any two ideals $\mathfrak{a}, \mathfrak{b}$ of $R$,

$$
\tau(\mathfrak{a} \mathfrak{b}) \subseteq \tau(\mathfrak{a}) \tau(\mathfrak{b}) .
$$

Proof. Let $T=R \hat{\otimes}_{k} R$, and let $\Delta: T \rightarrow R$ be the ring homomorphism sending $x \otimes y \in T$ to $x y \in R$. If we restrict the containment $\tau(\mathfrak{a} \mathfrak{b} T) \subseteq \tau(\mathfrak{a}) \tau(\mathfrak{b}) T$ in 
Proposition 4.4 by the diagonal map $\Delta: T \rightarrow R$, we immediately obtain

$$
\tau(\mathfrak{a} \mathfrak{b}) \subseteq \tau(\mathfrak{a} \mathfrak{b} T) R \subseteq \tau(\mathfrak{a}) \tau(\mathfrak{b})
$$

by virtue of Theorem 4.1 .

Remark 4.6. If $(R, \mathfrak{m})$ is not F-regular, then it is clear that the subadditivity breaks down for $\mathfrak{a}=\mathfrak{b}=R$. We do not know whether or not the subadditivity always holds in F-regular rings in general.

4.7. Toric case. In $\mathrm{How}$, Howald gave a combinatorial description of the multiplier ideal $\mathcal{J}(\mathfrak{a})$ of a monomial ideal $\mathfrak{a}$ in a polynomial ring over a field. We show that the ideal $\tau(\mathfrak{a})$ has a similar description in a more general situation, namely, $\mathfrak{a}$ is a toric ideal of a toric ring $R$ over a field $k$. Note that, in this case, the multiplier ideal $\mathcal{J}(\mathfrak{a})$ can be defined even if char $k=p>0$, because there exists a log resolution of $\mathfrak{a}$ in the toric category.

Let $M=\mathbb{Z}^{d}, N=\operatorname{Hom}_{\mathbb{Z}}(M, \mathbb{Z})$, and denote the duality pairing of $M_{\mathbb{R}}=M \otimes_{\mathbb{Z}} \mathbb{R}$ with $N_{\mathbb{R}}=N \otimes_{\mathbb{Z}} \mathbb{R}$ by $\langle\rangle:, M_{\mathbb{R}} \times N_{\mathbb{R}} \rightarrow \mathbb{R}$. Let $\sigma \subset N_{\mathbb{R}}$ be a strongly convex rational polyhedral cone, and denote $\sigma^{\vee}=\left\{m \in M_{\mathbb{R}} \mid\langle m, n\rangle \geq 0\right.$ for all $\left.n \in \sigma\right\}$ as usual. Let $R=k\left[\sigma^{\vee} \cap M\right]$ be the toric ring over a field $k$ defined by $\sigma$, that is, the subring of a polynomial ring $k\left[x_{1}, \ldots, x_{d}\right]$ generated as a $k$-algebra by monomials $x^{m}=x_{1}^{m_{1}} \cdots x_{d}^{m_{d}}$ with $m=\left(m_{1}, \ldots, m_{d}\right) \in \sigma^{\vee} \cap M$. Also, let $D_{1}, \ldots, D_{s}$ be the toric divisors of Spec $R$ corresponding to the primitive generators $n_{1}, \ldots, n_{s} \in N$ of $\sigma$, respectively. A toric ideal $\mathfrak{a} \subseteq R$ is an ideal of $R$ generated by monomials in $x_{1}, \ldots, x_{d}$. Let $\mathfrak{a} \subseteq R$ be a toric ideal and let $P=P(\mathfrak{a}) \subset M_{\mathbb{R}}$ be the Newton polygon of $\mathfrak{a}$, that is, the convex hull of $\left\{m \in M \mid x^{m} \in \mathfrak{a}\right\}$ in $M_{\mathbb{R}}$. We denote the relative interior of $P$ in $M_{\mathbb{R}}$ by $\operatorname{Int}(P)$.

Now assume that $R$ is $\mathbb{Q}$-Gorenstein. Then there exists $w \in M_{\mathbb{R}}$ such that $\left\langle w, n_{i}\right\rangle=1$ for $i=1, \ldots, s$. Indeed, since $\omega_{R}^{(r)}$ is principally generated for some $r \in \mathbb{N}$ and $\omega_{R}^{(r)}$ corresponds to the divisor $-r \sum_{i=1}^{s} D_{i}$, we can write $\omega_{R}^{(r)}=x^{m_{0}} R$ for some $m_{0} \in M$ such that $\left\langle m_{0}, n_{i}\right\rangle=v_{D_{i}}\left(x^{m_{0}}\right)=r$. Then set $w=m_{0} / r \in M_{\mathbb{R}}$.

Theorem 4.8. Let $R=k\left[\sigma^{\vee} \cap M\right]$ be a $\mathbb{Q}$-Gorenstein toric ring over a field of characteristic $p>0$, and let $w \in M_{\mathbb{R}}$ be as above. Then, for any toric ideal $\mathfrak{a} \subseteq R$,

$$
\tau(\mathfrak{a})=\mathcal{J}(\mathfrak{a}),
$$

and it is again a toric ideal. Moreover, for $m \in M$, the following conditions are equivalent to each other:

(1) $x^{m} \in \tau(\mathfrak{a})$;

(2) $m+w \in \operatorname{Int}(P(\mathfrak{a}))$;

(3) $x^{m} \in \mathcal{J}(\mathfrak{a})$.

Proof. We prove that $\tau(\mathfrak{a})$ is generated by monomials $x^{m}$ satisfying the condition $m+w \in \operatorname{Int}(P(\mathfrak{a}))$ in $(2)$. It is essentially proved in How that $\mathcal{J}(\mathfrak{a})$ has the same property.

First, to simplify our argument, we note that $1 \in R^{\circ}$ is an a-test element, because toric rings are strongly F-regular. Hence, an element $z \in E$ of the injective envelope $E=E_{R}(R / \mathfrak{m})$ of the residue field $R / \mathfrak{m}=k$ is in $0_{E}^{* \mathfrak{a}}$ if and only if $z^{q} \mathfrak{a}^{q}=0$ in $\mathbb{F}^{e}(E)={ }^{e} R \otimes_{R} E$ for all $q=p^{e}$.

Next we will compute the Frobenius map $F^{e}: E \rightarrow \mathbb{F}^{e}(E)$ explicitly. To do this we note that $\mathbb{F}^{e}(E) \cong H_{\mathfrak{m}}^{d}\left(\omega_{R}^{(q)}\right)$ for $q=p^{e}$ by $\left[\mathrm{Wa}\right.$, and $H_{\mathfrak{m}}^{d}\left(\omega_{R}^{(q)}\right)$ is $k$-dual to 
$\omega_{R}^{(1-q)}=\bigoplus_{\left\langle m, n_{i}\right\rangle \geq 1-q} k \cdot x^{m}$. Therefore,

$$
\mathbb{F}^{e}(E)=\bigoplus_{\left\langle m, n_{i}\right\rangle \leq q-1} k \cdot x^{m}=\bigoplus_{m \in(q-1) w-\sigma^{\vee}} k \cdot x^{m},
$$

and the Frobenius map $F^{e}: E \rightarrow \mathbb{F}^{e}(E)$ sends $x^{m} \in E$ to $x^{m q} \in \mathbb{F}^{e}(E)$.

It is now clear that $0_{E}^{* \mathfrak{a}}$ and hence $\tau(\mathfrak{a})=\operatorname{Ann}_{R}\left(0_{E}^{* \mathfrak{a}}\right)$ are generated by monomials, because everything involved is $\mathbb{Z}^{s}$-graded.

We describe the a-tight closure $0_{E}^{* \mathfrak{a}}$ of zero in $E=\bigoplus_{u \in-\sigma^{\vee} \cap M} k \cdot x^{u}$. Let $u \in-\sigma^{\vee} \cap M$. Then $x^{u} \in 0_{E}^{* \mathfrak{a}}$ if and only if $x^{q u} \mathfrak{a}^{q}=0$ in $\mathbb{F}^{e}(E)$ or, equivalently, $(q u+q P) \cap\left((q-1) w-\sigma^{\vee}\right) \cap M=\emptyset$, for all $q=p^{e}$. Dividing out by $q$, we can rephrase this into the condition that $(u+P) \cap \operatorname{Int}\left(w-\sigma^{\vee}\right)=\emptyset$, because $-w / q \in \operatorname{Int}\left(-\sigma^{\vee}\right)$. Since this is equivalent to saying that $\operatorname{Int}(u+P) \cap\left(w-\sigma^{\vee}\right)=\emptyset$, it follows that $x^{u} \in 0_{E}^{* \mathfrak{a}}$ if and only if $\operatorname{Int}(P) \cap\left(w-u-\sigma^{\vee}\right)=\emptyset$ or, equivalently, if $w-u \notin \operatorname{Int}(P)$.

Now the equivalence of conditions (1) and (2) follows immediately, because a monomial $x^{m} \in R=k\left[\sigma^{\vee} \cap M\right]$ is in $\tau(\mathfrak{a})=\operatorname{Ann}_{R}\left(0_{E}^{* \mathfrak{a}}\right)$ if and only if $x^{-m} \notin 0_{E}^{* \mathfrak{a}}$.

Example 4.9. Let $S=k\left[x_{1}, \ldots, x_{d}\right]$ be a polynomial ring and let $R=S^{(r)}$ be the $r$ th Veronese subring of $S$. We can easily compute the ideal $\tau(\mathfrak{a})=\mathcal{J}(\mathfrak{a})$ associated to a monomial ideal $\mathfrak{a}$ of $R$ as follows; cf. [How].

We choose $N$ and $M$ to be the overlattice $N=\mathbb{Z}^{d}+\frac{1}{r}(1, \ldots, 1) \mathbb{Z}$ and the sublattice $M=\left\{m \in \mathbb{Z}^{d} \mid\langle m, n\rangle \in \mathbb{Z}\right\}$ of $\mathbb{Z}^{d}$, respectively, and let $\sigma$ be the first orthant in $N_{\mathbb{R}}=\mathbb{R}^{d}$. Then the dual cone $\sigma^{\vee}$ is also the first orthant in $M_{\mathbb{R}}=\mathbb{R}^{d}$, and the ring $R=k\left[\sigma^{\vee} \cap M\right]$ is realized as it is as the $r$ th Veronese subring of $S=k\left[\sigma^{\vee} \cap \mathbb{Z}^{d}\right]=k\left[x_{1}, \ldots, x_{d}\right]$. In this setting, the vector $w \in M_{\mathbb{R}}$ (for both $R$ and $S$ ) defined in 4.7 is equal to $\mathbf{1}=(1,1, \ldots, 1)$. Also, for a monomial ideal $\mathfrak{a} \subseteq R$, the Newton polygons $P(\mathfrak{a})$ and $P(\mathfrak{a} S)$ are equal to each other in $M_{\mathbb{R}}=\mathbb{R}^{d}$. Therefore, Theorem 4.8 tells us that a monomial $x^{m}$ in $R$ (resp. $S$ ) is in $\tau(\mathfrak{a})(\operatorname{resp} . \tau(\mathfrak{a} S))$ if and only if $m+\mathbf{1} \in \operatorname{Int}(P(\mathfrak{a}))=\operatorname{Int}(P(\mathfrak{a} S))$, and, in particular,

$$
\tau(\mathfrak{a})=\tau(\mathfrak{a} S) \cap R ;
$$

cf. Lemma 3.6. For example, if $\mathfrak{a}=\mathfrak{m}^{l}$ is a power of the graded maximal ideal $\mathfrak{m}$ of $R$, we have $\tau\left(\mathfrak{m}^{l}\right)=\mathfrak{m}^{\lceil l-(d-1) / r\rceil}=\mathfrak{m}^{\lfloor l+1-d / r\rfloor}$.

\section{F-rationality of Rees algebras and the Behavior of $\tau(I)$}

Throughout this section, we assume that $(R, \mathfrak{m})$ is an excellent Gorenstein local domain of characteristic $p>0$, and that $I$ is an $\mathfrak{m}$-primary ideal of $R$. Put $d=$ $\operatorname{dim} R \geq 2$. Let $\mathbf{R}(I)=R[I t]$ denote the Rees algebra of $I$ over $R$, and $\mathfrak{M}=$ $\mathfrak{m} \mathbf{R}(I)+\mathbf{R}(I)_{+}$, the unique homogeneous maximal ideal of $\mathbf{R}(I)$. We will denote by $\mathbf{R}^{\prime}(I)=R\left[I t, t^{-1}\right]$ and $G(I)=\mathbf{R}^{\prime}(I) / t^{-1} \mathbf{R}^{\prime}(I)=\bigoplus_{n \geq 0} I^{n} / I^{n+1}$ the extended Rees algebra and the associated graded ring of $I$, respectively. Also, let $\omega_{\mathbf{R}(I)}$ denote the graded canonical module of $\mathbf{R}(I)$, and let $\pi: Y=\operatorname{Proj} \mathbf{R}(I) \rightarrow \operatorname{Spec} R$ be the blowing-up with respect to $I$.

The main purpose of this section is to describe $\omega_{\mathbf{R}(I)}$ in terms of $\tau\left(I^{n}\right)$ under the assumption that $\mathbf{R}(I)$ is F-rational. Actually, we prove the following theorem.

Theorem 5.1. Let $(R, \mathfrak{m})$ be an excellent Gorenstein local domain of characteristic $p>0$ with $d=\operatorname{dim} R \geq 2$. Let $I$ be an $\mathfrak{m}$-primary ideal of $R$ and $J$ its minimal 
reduction. Then $\tau(I) \subseteq J: I^{d-1}$. If we assume that $\mathbf{R}(I)$ is F-rational, then $\tau(I)=J: I^{d-1}$ and

$$
\omega_{\mathbf{R}(I)}=\bigoplus_{n \geq 1} H^{0}\left(Y, I^{n} \omega_{Y}\right) \cong \bigoplus_{n \geq 1} \tau\left(I^{n}\right)
$$

Discussion 5.2. The above theorem is motivated by Hyry's papers [Hy1], [Hy2]. For example, the description of $\omega_{\mathbf{R}(I)}$ in Theorem 5.1 corresponds to the following fact used in [Hy1]: Let $(R, \mathfrak{m})$ be a regular local ring essentially of finite type over a field of characteristic zero, and let $I$ be an ideal of $R$. Suppose that $\operatorname{Proj} \mathbf{R}(I)$ has rational singularities. The graded canonical module of $\mathbf{R}(I)$ is then $\omega_{\mathbf{R}(I)}=\bigoplus_{n>1} \mathcal{J}\left(I^{n}\right)$.

Actually, if $\mathbf{R}(I)$ is F-rational, then so is $Y=\operatorname{Proj} \mathbf{R}(I)$, whence $Y$ is pseudorational Sm1. Therefore, if $Y$ has a resolution of singularities $f: X \rightarrow Y$, then $H^{0}\left(X, I^{n} \omega_{X}\right) \cong H^{0}\left(Y, I^{n} \omega_{Y}\right)$ for every $n \geq 0$. The left-hand side of this equality coincides with the multiplier ideal $\mathcal{J}\left(I^{n}\right)$ via the isomorphism $\omega_{R} \cong R$ as long as $\mathcal{J}\left(I^{n}\right)$ is defined. Moreover, $\left[\omega_{\mathbf{R}(I)}\right]_{n} \cong H^{0}\left(Y, I^{n} \omega_{Y}\right)$, since $\mathbf{R}(I)$ is CohenMacaulay; see e.g. [HHK]. In particular, we have $\tau(I)=\mathcal{J}(I)$ in this case.

Consequently, Theorem 5.1 claims that the F-rationality of $\mathbf{R}(I)$ gives a sufficient condition for $\tau(I)=\mathcal{J}(I)$ to hold in any fixed positive characteristic.

We obtain the following corollary from Theorem 5.1 and HWY1 Corollary 3.3].

Corollary 5.3. Suppose that $(R, \mathfrak{m})$ is a two-dimensional rational double point. Let $I$ be an $\mathfrak{m}$-primary integrally closed ideal of $R$, and $J$ its minimal reduction. Then $\tau(I) \subseteq J: I(=\mathcal{J}(I))$. Also, $\mathbf{R}(I)$ is F-rational if and only if $\tau(I)=J: I$.

One can easily check the following example using the method developed in [HWY1 Section 3].

Example 5.4 (cf. [HWY1, Theorem 3.1]). (1) Let $(R, \mathfrak{m})$ be a two-dimensional excellent Gorenstein F-rational local ring (i.e., F-rational double point), and $I$ an m-primary integrally closed ideal of $R$. Then $\tau(I)=J: I$ for any minimal reduction $J$ of $I$.

(2) Let $R=k[[x, y, z]] /\left(x^{2}+y^{3}+z^{5}\right)$, where $k$ is an algebraically closed field of characteristic 2. Put $\mathfrak{m}=(x, y, z) R$ and $J=(y, z) R$. Then $R$ is a two-dimensional rational double point, but not F-rational. Also, we have:

(a) $R(\mathfrak{m})$ is not F-rational.

(b) $J^{* \mathfrak{m}}=\mathfrak{m}$. In particular, $J: J^{* \mathfrak{m}}=\mathfrak{m}$.

(c) $\left(J^{[2]}\right)^{* \mathfrak{m}}=\left(y^{2}, z^{2}, x y\right)$. In particular, $J^{[2]}:\left(J^{[2]}\right)^{* \mathfrak{m}}=\left(x, y, z^{2}\right)$.

(d) $\tau(\mathfrak{m}) \subseteq\left(x, y, z^{2}\right) \subsetneq \mathcal{J}(\mathfrak{m})=\mathfrak{m}$.

In the following, we will prove Theorem 5.1.

Lemma 5.5. Let $(R, \mathfrak{m})$ be a Gorenstein local ring of any characteristic. Also, let $I$ be an $\mathfrak{m}$-primary ideal of $R$ and put $G(I)=\bigoplus_{n \geq 0} I^{n} / I^{n+1}$, the associated graded ring of $I$. Assume that $\left[H_{\mathfrak{M}}^{d}(\mathbf{R}(I)]_{0}=\left[H_{\mathfrak{M}}^{d}(\mathbf{R}(I))\right]_{-1}=0\right.$ (e.g., $\mathbf{R}(I)$ is Cohen-Macaulay). Then $R /\left[\omega_{\mathbf{R}(I)}\right]_{1} \cong\left(\left[H_{\mathfrak{M}}^{d}(G(I))\right]_{-1}\right)^{\vee}$, where ()$^{\vee}$ denotes the Matlis dual of $R$.

Proof. Consider the following two standard exact sequences:

$$
\begin{aligned}
& 0 \longrightarrow \mathbf{R}(I)_{+} \longrightarrow \mathbf{R}(I) \longrightarrow R \longrightarrow 0, \\
& 0 \longrightarrow \mathbf{R}(I)_{+}(1) \longrightarrow \mathbf{R}(I) \longrightarrow G(I) \longrightarrow 0 .
\end{aligned}
$$


From the first exact sequence, we have

$$
0=\left[H_{\mathfrak{M}}^{d}(\mathbf{R}(I))\right]_{0} \rightarrow H_{\mathfrak{m}}^{d}(R) \rightarrow\left[H_{\mathfrak{M}}^{d+1}\left(\mathbf{R}(I)_{+}\right)\right]_{0} \rightarrow\left[H_{\mathfrak{M}}^{d+1}(\mathbf{R}(I))\right]_{0}=0,
$$

where the vanishing on the right follows from $a(\mathbf{R}(I))=-1$. Since $R$ is Gorenstein, $R=\omega_{R} \cong\left(H_{\mathfrak{m}}^{d}(R)\right)^{\vee} \cong\left(\left[H_{\mathfrak{M}}^{d+1}\left(\mathbf{R}(I)_{+}\right)\right]_{0}\right)^{\vee}$. On the other hand, the second exact sequence gives

$$
0=\left[H_{\mathfrak{M}}^{d}(\mathbf{R}(I))\right]_{-1} \rightarrow\left[H_{\mathfrak{M}}^{d}(G(I))\right]_{-1} \rightarrow\left[H_{\mathfrak{M}}^{d+1}\left(\mathbf{R}(I)_{+}\right)\right]_{0} \rightarrow\left[H_{\mathfrak{M}}^{d+1}(\mathbf{R}(I))\right]_{-1} \rightarrow 0 .
$$

Dualizing the above sequence, we get

$$
0 \longrightarrow\left[\omega_{\mathbf{R}(I)}\right]_{1} \longrightarrow\left(\left[H_{\mathfrak{M}}^{d+1}\left(\mathbf{R}(I)_{+}\right)\right]_{0}\right)^{\vee} \cong R \longrightarrow\left(\left[H_{\mathfrak{M}}^{d}(G(I))\right]_{-1}\right)^{\vee} \longrightarrow 0 .
$$

This yields the required assertion.

Proposition 5.6. Using the same notation as in Lemma 5.5, assume further that $\mathbf{R}(I)$ is Cohen-Macaulay. Then $\left[\omega_{\mathbf{R}(I)}\right]_{1}=J: I^{d-1}$ for every minimal reduction $J$ of $I$.

Proof. Let $x_{1}, x_{2}, \ldots, x_{d}$ be a minimal system of generators of $J$. Put $G:=G(I)$. Then it is well known that $G(I)$ is Cohen-Macaulay and the images in $G$ of $x_{1} t, \ldots, x_{d} t\left(\in \mathbf{R}(I)_{1}\right)$ form a regular sequence ([GS]). Setting $x_{i}^{*}:=x_{i} t \bmod I^{2}$ for each $i$, we have an exact sequence

$$
0 \longrightarrow G(-1) \stackrel{x_{1}^{*}}{\longrightarrow} G \longrightarrow G / x_{1}^{*} G \cong G\left(I / x_{1} R\right) \longrightarrow 0
$$

by [VV]. From this sequence, we get an exact sequence

$$
0=H_{\mathfrak{M}}^{d-1}(G) \longrightarrow H_{\mathfrak{M}}^{d-1}\left(G / x_{1}^{*} G\right) \longrightarrow H_{\mathfrak{M}}^{d}(G)(-1) \stackrel{x_{1}^{*}}{\longrightarrow} H_{\mathfrak{M}}^{d}(G) \longrightarrow 0 .
$$

Since $a(G) \leq-1([\mathrm{GS}])$, we have $\left[H_{\mathfrak{M}}^{d-1}\left(G / x_{1}^{*} G\right)\right]_{0} \cong\left[H_{\mathfrak{M}}^{d}(G)\right]_{-1}$ and $a\left(G / x_{1}^{*} G\right) \leq$ $-1+1=0$. By repeating the above argument, we get

$$
\left[H_{\mathfrak{M}}^{d}(G)\right]_{-1} \cong\left[H_{\mathfrak{M}}^{0}\left(G /\left(x_{1}^{*}, \ldots, x_{d}^{*}\right) G\right)\right]_{d-1} \cong\left[H_{\mathfrak{M}}^{0}(G(I / J))\right]_{d-1}=\frac{J+I^{d-1}}{J} .
$$

Also, since $R / J$ is Gorenstein, we have $\left(\frac{J+I^{d-1}}{J}\right)^{\vee} \cong R / J: I^{d-1}$. Combining this with the previous lemma, we get

$$
\left[\omega_{\mathbf{R}(I)}\right]_{1}=\operatorname{Ann}_{R}\left(\left[H_{\mathfrak{M}}^{d}(G)\right]_{-1}\right)^{\vee}=\operatorname{Ann}_{R}\left(\frac{J+I^{d-1}}{J}\right)^{\vee}=J: I^{d-1} .
$$

Proposition 5.7. Let $(R, \mathfrak{m})$ be an excellent Gorenstein local domain of characteristic $p>0$, and let $I$ be an $\mathfrak{m}$-primary ideal of $R$. Also, let $J$ be a minimal reduction of $I$. Then we have the following statements.

(1) $\tau(I) \subseteq J: J^{* I} \subseteq J: I^{d-1}$.

(2) If $\mathbf{R}(\bar{I})$ is F-rational, then $\tau(I)=J: J^{* I}=J: I^{d-1}$.

Proof. Let $x_{1}, \ldots, x_{d}$ be a system of generators of $J$ and put $J^{[l]}:=\left(x_{1}^{l}, \ldots, x_{d}^{l}\right)$ for all integers $l \geq 1$.

(1) One has $\tau(I) \subseteq J: J^{* I} \subseteq J:\left(J+I^{d-1}\right)=J: I^{d-1}$ by the definition of $\tau(I)$ and Corollary 2.8.

To see (2), we may assume that $\mathbf{R}(I)$ is Cohen-Macaulay. Then $I^{d}=J I^{d-1}$ (兽]). Hence $I^{d l-1}=J^{d l-d} I^{d-1}=\left(J^{[l]} J^{d l-d-l}+\left(x_{1} \cdots x_{d}\right)^{l-1} R\right) I^{d-1}$. Thus 
$J^{[l]}+I^{d l-1}=J^{[l]}+\left(x_{1} \cdots x_{d}\right)^{l-1} I^{d-1}$. In particular, for all $l \geq 1$, we have $J^{[l]}$ : $\left(J^{[l]}+I^{d l-1}\right)=\left(J^{[l]}:\left(x_{1} \cdots x_{d}\right)^{l-1}\right): I^{d-1}=J: I^{d-1}$.

Now suppose that $\mathbf{R}(I)$ is F-rational. Then since $\left(J^{[l]}\right)^{* I}=J^{[l]}+I^{d l-1}$ by Corollary 2.9, we have that $J^{[l]}:\left(J^{[l]}\right)^{* I}=J: I^{d-1}$ for all $l \geq 1$. Hence $\tau(I)=$ $J: J^{* I}=J: I^{d-1}$, as required.

Proof of Theorem 5.1. Note that $\mathbf{R}\left(I^{n}\right)$ is F-rational if $\mathbf{R}(I)$ is. Actually, it is a module-finite pure subring of $\mathbf{R}(I)$. Thus the required assertion immediately follows from Propositions 5.6 and 5.7.

Proof of Corollary 5.3. Let $I$ be an m-primary integrally closed ideal and $J$ its minimal reduction. Then it is well known that $I^{2}=J I$, and thus $\mathbf{R}(I)$ is CohenMacaulay.

It is enough to show that $\tau(I)=J: I$ implies that $\mathbf{R}(I)$ is F-rational. Suppose that $\tau(I)=J: I$. Since $\tau(I) \subseteq J^{[l]}:\left(J^{[l]}\right)^{* I} \subseteq J^{[l]}:\left(J^{[l]}+I^{2 l-1}\right)=J: I$, in general, we have $J^{[l]}:\left(J^{[l]}\right)^{* I}=J^{[l]}:\left(J^{[l]}+I^{2 l-1}\right)$. This implies that $\left(J^{[l]}\right)^{* I}=J^{[l]}+I^{2 l-1}$ for all $l \geq 1$, because $R / J^{[l]}$ is an Artinian Gorenstein local ring. By [HWY1, Corollary 3.3(2)], we conclude that $\mathbf{R}(I)$ is F-rational.

In the rest of this section, we will give some applications of Theorem 5.1. Before stating our results, let us recall the notion of $a$-invariant. Let $I$ be an $\mathfrak{m}$-primary ideal of $R$, and put $G:=G(I)$ and $\mathfrak{M}:=\mathfrak{m} \mathbf{R}(I)+\mathbf{R}(I)_{+}$. Then the $a$-invariant $a(G)$ of $G$ is defined by $a(G):=\max \left\{n \in \mathbb{Z} \mid\left[H_{\mathfrak{M}}^{d}(G)\right]_{n} \neq 0\right\}$. See [GW] for details.

Proposition 5.8. Let $(R, \mathfrak{m})$ be an excellent Gorenstein local domain of characteristic $p>0$. Let $I$ be an $\mathfrak{m}$-primary ideal of $R$. Suppose that $\mathbf{R}(I)$ is F-rational and $G:=G(I)$ is Gorenstein. Then $\tau\left(I^{n}\right)=I^{n+a(G)+1}$ for all integers $n \geq 1$.

Proof. The F-rationality of $\mathbf{R}(I)$ implies that $\tau\left(I^{n}\right)=\left[\omega_{\mathbf{R}(I)}\right]_{n}=H^{0}\left(Y, I^{n} \omega_{Y}\right)$ for all $n \geq 1$; see Discussion 5.2. Also, since $G$ is Cohen-Macaulay, we have

$$
\omega_{G} \cong \bigoplus_{n \geq 1} H^{0}\left(Y, I^{n-1} \omega_{Y}\right) / H^{0}\left(Y, I^{n} \omega_{Y}\right)
$$

and $R=H^{0}\left(Y, \omega_{Y}\right)=\cdots=H^{0}\left(Y, I^{-a-1} \omega_{Y}\right)$, where $a=a(G) \leq-1$; see e.g. Hy2. Theorem 2.2]. On the other hand, since $G$ is Gorenstein, we have

$$
\omega_{G} \cong G(a)=\bigoplus_{n \geq-a} I^{n+a} / I^{n+a+1}
$$

Comparing (5.8.1) with (5.8.2), one can easily see that $\tau\left(I^{n}\right)=I^{n+a+1}$ by induction on $n \geq 1$.

Corollary 5.9. Let $(R, \mathfrak{m})$ be an (excellent) regular local ring of characteristic $p>0$. Then $\tau\left(\mathfrak{m}^{n}\right)=\mathfrak{m}^{n-d+1}$ for all $n \geq 1$.

Proof. Suppose that $R$ is a regular local ring. Then $R(\mathfrak{m})$ is F-rational and $G(\mathfrak{m}) \cong$ $k\left[X_{1}, \ldots, X_{d}\right]$ is Gorenstein with $a(G(\mathfrak{m}))=-d$. Hence we can apply the above proposition.

Remark 5.10. Corollary 5.9 is a generalization of the implication $(1) \Rightarrow(2)$ in Theorem 2.15. This also follows from Theorem 4.8.

Let $J \subseteq I$ be ideals of $R$. Recall that the coefficient ideal of $I$ relative to $J$, denoted by $\mathfrak{a}(I, J)$, is defined to be the largest ideal $\mathfrak{a}$ of $R$ for which $I \mathfrak{a}=J \mathfrak{a}$. 
Remark 5.11. In [Hy2], Hyry proved that if $R$ is a Gorenstein local ring and $\mathbf{R}(I)$ has rational singularities, then $\mathcal{J}\left(I^{d-1}\right)=\mathfrak{a}(I, J)$. In fact, a similar result follows from Theorem 5.1 and $[\mathrm{Hy2}$, Theorem 3.4]: Let $(R, \mathfrak{m})$ be an excellent Gorenstein local domain of characteristic $p>0$. Let $I$ be an $\mathfrak{m}$-primary ideal of $R$, and $J$ its minimal reduction. If $\mathbf{R}(I)$ is F-rational, then $\tau\left(I^{d-1}\right)=H^{0}\left(Y, I^{d-1} \omega_{Y}\right)=\mathfrak{a}(I, J)$. In particular, if, in addition, $I^{2}=J I$, then $\tau\left(I^{d-1}\right)=J: I$.

In the rest of this section, we direct our attention to the ideal $\tau(\mathfrak{m})$. Let $(R, \mathfrak{m})$ be an excellent Gorenstein F-rational local domain of characteristic $p>0$. Then $\tau(\mathfrak{m}) \supseteq \mathfrak{m}$, that is, $\tau(\mathfrak{m})=\mathfrak{m}$ or $\tau(\mathfrak{m})=R$. For example, if $R$ is a regular local ring with $\operatorname{dim} R \geq 2$, then $\tau(\mathfrak{m})=R$. More generally, we have the following proposition.

Proposition 5.12. Let $(R, \mathfrak{m})$ be an excellent Gorenstein local domain of characteristic $p>0$ with $d=\operatorname{dim} R \geq 2$. Suppose that there exists an $\mathfrak{m}$-primary ideal I such that $\mathbf{R}(I)$ is F-rational with $a(G(I)) \neq-1$. Then $R$ is F-rational with $\tau(\mathfrak{m})=R$.

Proof. By virtue of [HWY1, Corollary 2.13], $R$ is F-rational.

By Theorem 5.1, we have $\tau(I)=J: I^{d-1}$ for any minimal reduction $J$ of $I$. Since $\mathbf{R}(I)$ is Cohen-Macaulay with $a(G(I)) \neq-1$, we have that $I^{d-1}=J I^{d-2} \subseteq J$. Hence $\tau(I)=R$. In particular, $\tau(\mathfrak{m})=R$, because $\tau(\mathfrak{m}) \supseteq \tau(I)$.

In view of the above proposition it is natural to ask the following question.

Question 5.13. Let $(R, \mathfrak{m})$ be an excellent Gorenstein F-rational local domain of characteristic $p>0$ with $\tau(\mathfrak{m})=R$ and $\operatorname{dim} R \geq 2$. When is $\mathbf{R}(\mathfrak{m})$ F-rational then?

In the case of two-dimensional local rings, $\tau(\mathfrak{m})=R$ implies that $R$ is regular. Then $R(\mathfrak{m})$ is Gorenstein and F-rational with $a(G(\mathfrak{m}))=-2$. As for threedimensional local rings, we have the following answer to the above question.

Proposition 5.14. Let $(R, \mathfrak{m})$ be a three-dimensional excellent Gorenstein local ring that is not regular. Then the following conditions are equivalent.

(1) $\tau(\mathfrak{m})=R$, that is, $I^{* \mathfrak{m}}=I$ holds for every ideal $I$ of $R$.

(2) $J^{* \mathfrak{m}}=J$ holds for some parameter ideal $J$ of $R$.

(3) $\mathbf{R}^{\prime}(\mathfrak{m})$ is F-rational, and $\mathfrak{m}^{2}=J \mathfrak{m}$ for some minimal reduction $J$ of $\mathfrak{m}$.

(4) $\mathbf{R}(\mathfrak{m})$ is Gorenstein and F-rational.

(5) $\tau\left(\mathfrak{m}^{n}\right)=\mathfrak{m}^{n-1}$ holds for all integers $n \geq 1$.

Proof. (1) $\Rightarrow(2)$ and $(5) \Rightarrow(1)$ are trivial. (4) $\Rightarrow(5)$ follows from Proposition 5.8, since $a(G(\mathfrak{m}))=-2([\underline{G S})$.

To see that $(2) \Rightarrow(3)$, we may assume that $J$ is a minimal reduction of $\mathfrak{m}$; see Discussion 1.14. By Corollary 2.8, we have $\mathfrak{m}^{2} \subseteq J$, and thus $\mathfrak{m}^{2}=J \mathfrak{m}$. Also, $\mathbf{R}^{\prime}(\mathfrak{m})$ is F-rational by [HWY1, Corollary 4.5].

$(3) \Rightarrow(4)$ : Note that a Gorenstein local ring having minimal multiplicity is a hypersurface with multiplicity at most 2 . Thus $R$ and $G(\mathfrak{m})$ are hypersurfaces, and $a(G(\mathfrak{m}))=1-\operatorname{dim} R=-2$. Hence $\mathbf{R}(\mathfrak{m})$ is Gorenstein (GS]). Also, since $\mathbf{R}^{\prime}(\mathfrak{m})$ is Gorenstein and F-rational, $\mathbf{R}(\mathfrak{m})$ is F-regular.

Discussion 5.15. We can generalize the equivalence of (2) and (3) in Proposition 5.14 as follows; see also Theorem 2.15 .

Let $(R, \mathfrak{m})$ be an excellent equidimensional reduced local ring of characteristic $p>0$. Then the following conditions are equivalent. 
(1) $\mathbf{R}^{\prime}(\mathfrak{m})$ is F-rational, and $\mathfrak{m}^{2}=J \mathfrak{m}$ for some minimal reduction $J$ of $\mathfrak{m}$.

(2) $J^{* \mathfrak{m}^{d-2}}=J$ holds for every (or equivalently, some) parameter ideal $J$ of $A$.

If, in addition, $R$ is Gorenstein, then the following condition is also equivalent to the above conditions:

(3) $\tau\left(\mathfrak{m}^{d-2}\right)=R$.

Example 5.16 (cf. [HWY2, Proposition 3.12], [HWY1, Sect. 5]). Let $(R, \mathfrak{m})$ be an excellent three-dimensional Gorenstein normal local domain of characteristic $p>0$. If $R$ admits a non-zero-divisor $f \in \mathfrak{m}$ such that $R / f R$ is F-rational, then $\tau(\mathfrak{m})=R$.

For example, let $R=k[[x, y, z, w]] /\left(x^{2}+y^{a}+z^{b}+w^{c}\right)$, where $k$ is a field of characteristic $p>0$, and $a, b, c$ are integers with $2 \leq a \leq b \leq c \ll p$. If $1 / 2+1 / a+$ $1 / b>1$, then $\tau(\mathfrak{m})=R$. Otherwise, $\tau(\mathfrak{m})=\mathfrak{m}$.

Remark 5.17. (1) If $R$ is a three-dimensional regular local ring, then $\tau\left(\mathfrak{m}^{2}\right)=R$ (and thus $\tau(\mathfrak{m})=R$ ) and $\mathbf{R}(\mathfrak{m})$ is F-rational. But $\mathbf{R}(\mathfrak{m})$ is not Gorenstein, and $\tau\left(\mathfrak{m}^{n}\right)=\mathfrak{m}^{n-2}$ for all $n \geq 1$.

(2) We have no examples of a Gorenstein local ring $R$ for which $\tau(\mathfrak{m})=R$ but $\mathbf{R}(\mathfrak{m})$ is not F-rational.

Discussion 5.18. Let $(R, \mathfrak{m})$ be a complete regular local ring of characteristic $p>0$ with $d=\operatorname{dim} R \geq 2$, and let $I$ be an $\mathfrak{m}$-primary ideal of $R$. Then we expect that $\tau(I) \supsetneq I$.

For example, this is true if $R(I)$ is F-rational. We sketch a proof here. Suppose that $\tau(I)=I$. Then $\tau\left(I^{n}\right) \subseteq \tau(I)^{n}=I^{n}$ for all $n \geq 1$, by the subadditivity (Theorem 4.5). On the other hand, since $R$ is F-regular, we have $\tau\left(I^{n}\right) \supseteq I^{n}$ in general. Also, by Theorem 5.1, we have $\omega_{\mathbf{R}(I)}=\bigoplus_{n \geq 1} I^{n}=\mathbf{R}(I)_{+}$. In particular, since $\mathbf{R}(I) / \omega_{\mathbf{R}(I)} \cong R$ is regular, so is $\mathbf{R}(I)$. (Note: Recently, S. Goto et. al. proved a more general result.) But this is impossible, because $\operatorname{dim} R \geq 2$. Hence $\tau(I) \supsetneq I$, as required.

As for multiplier ideals, the authors are informed of the following result by K.i. Watanabe: Let $R$ be a regular local ring essentially of finite type over a field of characteristic zero. Then $\mathcal{J}(I) \supsetneq I$ for any $\mathfrak{m}$-primary ideal $I$ of $R$.

\section{Rational CoefFicients}

Recently, the theory of multiplier ideals with "rational coefficients" has been developed and applied successfully to various problems in algebraic geometry and commutative algebra ([ELS], La $)$. This motivates us to extend the notions of atight closure and the ideal $\tau(\mathfrak{a})$ to those with "rational coefficients". In this last section we make a few remarks on rational coefficients and address the results that generalize in this setting.

Definition 6.1. Let $\mathfrak{a}$ be an ideal of a Noetherian ring $R$ of characteristic $p>0$ such that $\mathfrak{a} \cap R^{\circ} \neq \emptyset$, and let $N \subseteq M$ be $R$-modules. Given a rational number $t \geq 0$, the $t \cdot \mathfrak{a}$-tight closure $N_{M}^{* t \cdot \mathfrak{a}}$ (or, $\mathfrak{a}^{t}$-tight closure $N_{M}^{* \mathfrak{a}}{ }^{t}$, see Remark 6.2 (1) below) of $N$ in $M$ is defined to be the submodule of $M$ consisting of all elements $z \in M$ for which there exists $c \in R^{\circ}$ such that

$$
c z^{q} \mathfrak{a}^{\lceil t q\rceil} \subseteq N_{M}^{[q]}
$$


for all $q=p^{e} \gg 0$, where $\lceil t q\rceil$ denotes the least integer that is greater than or equal to $t q$.

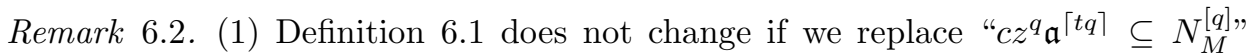

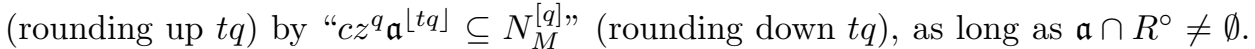
This is because the difference of $\lceil t q\rceil$ and $\lfloor t q\rfloor$ as the exponents of $\mathfrak{a}$ is "absorbed" by the term $c \in R^{\circ}$. Similarly, it is easy to see that $t \cdot \mathfrak{a}^{n}$-tight closure is the same as $t n \cdot \mathfrak{a}$-tight closure for every nonnegative integer $n$; cf. the proof of $[\mathrm{HW}$, Proposition 2.6]. This being so, it is preferable to say " $\mathfrak{a}$-tight closure" rather than " $t \cdot \mathfrak{a}$-tight closure." In the sequel, we always use "exponential notation" in this manner and denote the $\mathfrak{a}^{t}$-tight closure of $N$ in $M$ by $N_{M}^{* \mathfrak{a}^{t}}$.

(2) The above formulation extends to several rational coefficients (or, several rational exponents). Namely, given ideals $\mathfrak{a}_{1}, \ldots, \mathfrak{a}_{r} \subseteq R$ with $\mathfrak{a}_{i} \cap R^{\circ} \neq \emptyset$ and rational numbers $t_{1}, \ldots, t_{r} \geq 0$, if $t_{i}=t n_{i}$ for nonnegative $t \in \mathbb{Q}$ and $n_{i} \in \mathbb{Z}$ with $i=1, \ldots, r$, we can define $\mathfrak{a}_{1}^{t_{1}} \cdots \mathfrak{a}_{r}^{t_{r}}$-tight closure to be $\left(\mathfrak{a}_{1}^{n_{1}} \cdots \mathfrak{a}_{r}^{n_{r}}\right)^{t}$-tight closure. If $N$ is a submodule of an $R$-module $M$, then an element $z \in M$ is in the $\mathfrak{a}_{1}^{t_{1}} \cdots \mathfrak{a}_{r}^{t_{r}}$ tight closure $N_{M}^{* \mathfrak{a}_{1}^{t_{1}} \cdots \mathfrak{a}_{r}^{t_{r}}}$ of $N$ in $M$ if and only if there exists $c \in R^{\circ}$ such that $c z^{q} \mathfrak{a}_{1}^{\left\lceil t_{1} q\right\rceil} \ldots \mathfrak{a}_{r}^{\left\lceil t_{r} q\right\rceil} \subseteq N_{M}^{[q]}$ for all $q=p^{e} \gg 0$.

(3) We also have an analogous notion of $\Delta$-tight closure for a pair $(R, \Delta)$ of a normal ring $R$ and a $\mathbb{Q}$-Weil divisor $\Delta$ on $Y=\operatorname{Spec} R$; see [T], [HW]. If $\mathfrak{a}_{i}=x_{i} R$ and $\Delta=\sum_{i=1}^{n} t_{i} \cdot \operatorname{div}_{Y}\left(x_{i}\right)$ for $x_{i} \in R$ and $0 \leq t_{i} \in \mathbb{Q}$ with $1 \leq i \leq n$, then $\mathfrak{a}_{1}^{t_{1}} \cdots \mathfrak{a}_{n}^{t_{n}}$-tight closure is the same as $\Delta$-tight closure.

Definition 6.3. Let $\mathfrak{a}$ be an ideal of a Noetherian $\operatorname{ring} R$ of characteristic $p>0$ such that $\mathfrak{a} \cap R^{\circ} \neq \emptyset$, and let $t \geq 0$ be a rational number. We say that an element $c \in R^{\circ}$ is an $\mathfrak{a}^{t}$-test element if $c z^{q} \mathfrak{a}^{\lceil t q\rceil} \subseteq I^{[q]}$ for all $q=p^{e}$ whenever $z \in I^{* \mathfrak{a}^{t}}$.

Some results for $\mathfrak{a}$-tight closure generalize to those for $\mathfrak{a}^{t}$-tight closure without essential change of proofs. However, we must be careful about the difference of round-up and round-down when speaking of $\mathfrak{a}^{t}$-test elements. As a matter of fact, the following theorem is proved similarly to Theorem $1.7(1)$, but the proof does not work if we replace $\lceil t q\rceil$ by $\lfloor t q\rfloor$ in Definition 6.3 .

Theorem 6.4. Let $R$ be an F-finite reduced ring of characteristic $p>0$ and let $c \in R^{\circ}$ be an element such that the localized ring $R_{c}$ is strongly F-regular. Then some power $c^{n}$ of $c$ is an $\mathfrak{a}^{t}$-test element for all ideals $\mathfrak{a} \subseteq R$ with $\mathfrak{a} \cap R^{\circ} \neq \emptyset$ and all rational numbers $t \geq 0$.

We can define the ideal $\tau\left(\mathfrak{a}^{t}\right)$ in a similar way to Proposition-Definition 1.9. Also, Theorem 1.13 generalizes to the case of $\tau\left(\mathfrak{a}^{t}\right)$ with the same proof. We summarize the results for excellent reduced local rings in the following.

Definition-Theorem 6.5. Let $(R, \mathfrak{m})$ be an excellent reduced local ring of characteristic $p>0$ with $E=E_{R}(R / \mathfrak{m})$, and let $\mathfrak{a} \subseteq R$ be an ideal such that $\mathfrak{a} \cap R^{\circ} \neq \emptyset$. Given a rational number $t \geq 0$, we define the ideal $\tau\left(\mathfrak{a}^{t}\right) \subseteq R$ by

$$
\tau\left(\mathfrak{a}^{t}\right)=\bigcap_{M} \operatorname{Ann}_{R}\left(0_{M}^{* \mathfrak{a}^{t}}\right)=\bigcap_{M \subseteq E} \operatorname{Ann}_{R}\left(0_{M}^{* \mathfrak{a}^{t}}\right)=\bigcap_{I \subseteq R}\left(I: I^{* \mathfrak{a}^{t}}\right),
$$

where $M$ runs through all finitely generated $R$-modules (resp. finitely generated $R$ submodules of $E$ ) in the second term (resp. the third term), and I runs through all 
ideals of $R$. Moreover, if $R$ is normal and $\mathbb{Q}$-Gorenstein, then

$$
\tau\left(\mathfrak{a}^{t}\right)=\operatorname{Ann}_{R}\left(0_{E}^{* \mathfrak{a}^{t}}\right) .
$$

Remark 6.6. We can define the ideal $\tau\left(\mathfrak{a}_{1}^{t_{1}} \cdots \mathfrak{a}_{n}^{t_{n}}\right)$ with several rational coefficients by replacing $\mathfrak{a}^{t}$-tight closure in 6.5 by $\mathfrak{a}_{1}^{t_{1}} \cdots \mathfrak{a}_{n}^{t_{n}}$-tight closure as defined in Remark 6.2 (2). See Theorem $6.10(2)$.

Proposition 1.15 also generalizes without essential change of the proof, but we cannot replace the round-up $\lceil t q\rceil$ by the round-down $\lfloor t q\rfloor$ in the following.

Proposition 6.7. Let $(R, \mathfrak{m})$ be a d-dimensional excellent normal local ring of characteristic $p>0, \mathfrak{a} \subseteq R$ an ideal such that $\mathfrak{a} \cap R^{\circ} \neq \emptyset$, and let $t \geq 0$ be a rational number. Then $0_{H_{\mathrm{m}}^{d}(R)}^{* \mathfrak{a}^{t}}$ is the unique maximal proper submodule $N$ with respect to the property

$$
\mathfrak{a}^{\lceil t q\rceil} F^{e}(N) \subseteq N \text { for all } q=p^{e},
$$

where $F^{e}: H_{\mathfrak{m}}^{d}(R) \rightarrow H_{\mathfrak{m}}^{d}(R)$ is the e-times iterated Frobenius induced on $H_{\mathfrak{m}}^{d}(R)$.

Now we generalize Theorem 3.4, which is the main theorem of Section 3, to the case of rational coefficients.

Theorem 6.8. Let $t \geq 0$ be a fixed rational number, $R$ a normal $\mathbb{Q}$-Gorenstein local ring essentially of finite type over a field, and let $\mathfrak{a}$ be a nonzero ideal. Assume that $\mathfrak{a} \subseteq R$ is reduced from characteristic zero to characteristic $p \gg 0$, together with a $\log$ resolution $f: X \rightarrow Y=\operatorname{Spec} R$ of the ideal a such that $\mathfrak{a} \mathcal{O}_{X}=\mathcal{O}_{X}(-Z)$. Then

$$
\tau\left(\mathfrak{a}^{t}\right)=H^{0}\left(X, \mathcal{O}_{X}\left(\left\lceil K_{X / Y}-t Z\right\rceil\right)\right) .
$$

Sketch of the proof. This is also proved in a similar way to Theorem 3.4. So we just indicate the points where some modification is needed in the following. First, we note that Lemma 3.6 holds for rational coefficients without changing the proof, i.e., $\tau\left((\mathfrak{a} S)^{t}\right) \cap R=\tau\left(\mathfrak{a}^{t}\right)$ under the assumption of Lemma 3.6, and that Lemma 3.7 is already proved for rational coefficients. Hence we can use a canonical covering of $R$ to reduce the proof of Theorem 6.8 to the quasi-Gorenstein case. Then it is sufficient to prove the following generalization of Theorem 3.9.

Theorem 6.9. Let $t \geq 0$ be a fixed rational number, $(R, \mathfrak{m})$ a d-dimensional normal local ring essentially of finite type over a field, and let $\mathfrak{a}$ be a nonzero ideal. Assume that $\mathfrak{a} \subseteq R$ is reduced from characteristic zero to characteristic $p \gg 0$, together with a $\log$ resolution $f: X \rightarrow Y=\operatorname{Spec} R$ of $\mathfrak{a}$ such that $\mathfrak{a} \mathcal{O}_{X}=\mathcal{O}_{X}(-Z)$. Then

$$
0_{H_{\mathfrak{m}}^{d}(R)}^{* \mathfrak{a}^{t}}=\operatorname{Ker}\left(H_{\mathfrak{m}}^{d}(R) \stackrel{\delta}{\longrightarrow} H_{E}^{d}\left(\mathcal{O}_{X}(t Z)\right)\right),
$$

where $E$ is the closed fiber of $f$ and $\delta$ is an edge map as in Proposition 3.8.

Here we note that the canonical dual of the sheaf $\mathcal{O}_{X}(t Z)=\mathcal{O}_{X}(\lfloor t Z\rfloor)$ is $\omega_{X}(-\lfloor t Z\rfloor)=\omega_{X}(\lceil-t Z\rceil)$, which is isomorphic to $\mathcal{O}_{X}\left(\left\lceil K_{X / R}-t Z\right\rceil\right)$ via $\omega_{R} \cong R$ if $R$ is quasi-Gorenstein.

The inclusion $0_{H_{\mathfrak{m}}^{d}(R)}^{* \mathfrak{a}^{t}} \supseteq \operatorname{Ker} \delta$ of the above theorem holds true in arbitrary fixed characteristic $p>0$ : Just take an element $c$ in the proof of Proposition 3.8 from $\mathfrak{a}^{\lceil t q\rceil} \subseteq H^{0}\left(X, \mathcal{O}_{X}(-t q Z)\right)$ instead of $\mathfrak{a}^{q}=H^{0}\left(X, \mathcal{O}_{X}(-q Z)\right)$, which gives rise to a map $c F^{e}: H_{E}^{d}\left(\mathcal{O}_{X}(t Z)\right) \rightarrow H_{E}^{d}\left(\mathcal{O}_{X}(t Z)\right)$. Then one sees that $\mathfrak{a}^{\lceil t q\rceil} F^{e}(\operatorname{Ker} \delta) \subseteq$ Ker $\delta$ for all $q=p^{e}$, and Proposition 6.7 applies. 
To prove the reverse inclusion $0_{H_{\mathrm{m}}^{d}(R)}^{* \mathfrak{a}^{t}} \subseteq \operatorname{Ker} \delta$, we choose, in characteristic zero before reducing to characteristic $p \gg 0$, a nonzero element $c \in \mathfrak{a}$ such that $R_{c}$ is regular and a $\log$ resolution $f: X \rightarrow$ Spec $R$ of the ideal $c \mathfrak{a}$, as in the proof of Theorem 3.9. Then choose an $f$-ample $f$-exceptional $\mathbb{Q}$-Cartier divisor $D$ and a sufficiently small $\varepsilon>0$ so that $\left\lfloor\tilde{Z}+\varepsilon \operatorname{div}_{X}(c)\right\rfloor=\lfloor t Z\rfloor$, where $\tilde{Z}=t Z-D$. We then move to reduction modulo $p \gg 0$ and let $m=r+2 s+\left\lceil n_{0} t\right\rceil$, keeping the integers $r, s, n_{0}$ just the same as in the proof of Theorem 3.9, i.e., the ideal $\mathfrak{a}$ is generated by $r$ elements, $c^{s}$ is a usual test element and also an $\mathfrak{a}^{t}$-test element, and $\mathcal{K}=\bigoplus_{n>0} H^{0}\left(X, \omega_{X}(-\lfloor n \tilde{Z}\rfloor)\right)$ is generated in degree $\leq n_{0}$ as a graded module over $\mathcal{R}=\bigoplus_{n \geq 0} H^{0}\left(X, \mathcal{O}_{X}(-n \tilde{Z})\right)$. Now, arguing as before, we obtain the required inclusion $0_{H_{\mathrm{m}}^{d}(R)}^{* \mathfrak{a}^{t}} \subseteq \operatorname{Ker} \delta$.

Finally, we note that the results in Section 4 also generalize to rational coefficients, with the same proof; see [DEL], [How, La] for the corresponding results for multiplier ideals.

Theorem 6.10. Let $t, t^{\prime}$ be any nonnegative rational numbers.

(1) (Restriction theorem): Under the assumption of Theorem 4.1 we have

$$
\tau\left((\mathfrak{a} S)^{t}\right) \subseteq \tau\left(\mathfrak{a}^{t}\right) S
$$

(2) (Subadditivity in regular local rings; cf. Remark 6.6): Under the assumption of Theorem 4.5 we have

$$
\tau\left(\mathfrak{a}^{t} \mathfrak{b}^{t^{\prime}}\right) \subseteq \tau\left(\mathfrak{a}^{t}\right) \tau\left(\mathfrak{b}^{t^{\prime}}\right)
$$

(3) Under the assumption of Theorem 4.8 , let $\mathfrak{a} \subseteq R$ be a toric ideal. Then $\tau\left(\mathfrak{a}^{t}\right)=\mathcal{J}\left(\mathfrak{a}^{t}\right)$, and it is also a toric ideal generated by monomials $x^{m}$ with $m \in M$ such that

$$
m+w \in \operatorname{Int}(t \cdot P(\mathfrak{a}))
$$

\section{REFERENCES}

$[\mathrm{AKM}]$ I. Aberbach, M. Katzman, and B. MacCrimmon, Weak F-regularity deforms in $\mathbb{Q}$ Gorenstein rings, J. Algebra 204 (1998), 281-285. MR 99d:13003

$[\mathrm{AM}] \quad$ I. Aberbach and B. MacCrimmon, Some results on test ideals, Proc. Edinburgh Math. Soc. (2) 42 (1999), 541-549. MR 2000i:13005

[B] J.-F. Boutot, Singularités rationnelles et quotients par les groupes réductifs, Invent. Math. 88 (1987), 65-68. MR 88a:14005

[BS] H. Skoda and J. Briançon, Sur la clôture intégrale d'un idéal de germes de fonctions holomorphes en un point de $C^{n}$, C. R. Acad. Sci. Paris Sér. A 278 (1974), 949-951. MR 49:5394

[BH] W. Bruns and J. Herzog, Cohen-Macaulay Rings, Cambridge Studies in Advanced Mathematics, vol. 39, Cambridge University Press, Cambridge, 1993. MR 95h:13020

[DEL] J.-P. Demailly, L. Ein and R. Lazarsfeld, A subadditivity property of multiplier ideals, Michigan Math. J. 48 (2000), 137-156. MR 2002a:14016

[Ei] L. Ein, Multiplier ideals, vanishing theorems and applications, in Algebraic GeometrySanta Cruz 1995, pp. 203-219, Proc. Sympos. Pure Math., vol. 62, American Mathematical Society, Providence, RI, 1997. MR 98m:14006

[ELS] L. Ein, R. Lazarsfeld and K. E. Smith, Uniform bounds and symbolic powers on smooth varieties, Invent. Math. 144 (2001), 241-252. MR 2002b:13001

[FW] R. Fedder and K.-i. Watanabe, A characterization of F-regularity in terms of F-purity, in Commutative Algebra, Berkeley 1987, pp. 227-245, Math. Sci. Res. Inst. Publ., vol. 15, Springer-Verlag, New York, 1989. MR 91k:13009 
[GS] S. Goto and Y. Shimoda, On the Rees algebras of Cohen-Macaulay local rings, in Commutative Algebra, Fairfax 1979, pp. 201-231, Lecture Notes in Pure and Appl. Math., vol. 68, Dekker, New York, 1982. MR 84a:13021

[GW] S. Goto and K.-i. Watanabe, On graded rings, I, J. Math. Soc. Japan 30 (1978), 179-213. MR 81m:13021

[Ha1] N. Hara, A characterization of rational singularities in terms of injectivity of Frobenius maps, Amer. J. Math. 120 (1998), 981-996. MR 99h:13005

[Ha2] Geometric interpretation of tight closure and test ideals, Trans. Amer. Math. Soc. 353 (2001), 1885-1906. MR 2001m:13009

[HT] N. Hara and S. Takagi, Some remarks on a generalization of test ideals, preprint.

[HW] N. Hara and K.-i. Watanabe, F-regular and F-pure rings vs. log terminal and log canonical singularities, J. Algebraic Geometry 11 (2002), 363-392. MR 2002k:13009

[HWY1] N. Hara, K.-i. Watanabe, and K. Yoshida, F-rationality of Rees algebras, J. Algebra 247 (2002), 153-190.

[HWY2] , Rees algebras of F-regular type, J. Algebra 247 (2002), 191-218.

[HHK] M. Herrmann, E. Hyry and T. Korb, On Rees algebras with a Gorenstein Veronese subring, J. Algebra 200 (1998), 279-311. MR 98m:13006

[Ho1] M. Hochster, Cyclic purity versus purity in excellent Noetherian rings, Trans. Amer. Math. Soc. 231 (1977), 463-488. MR 57:3111

[Ho2] M. Hochster, The tight integral closure of a set of ideals, J. Algebra 230 (2000), 184-203. MR 2002f: 13009

[HH0] M. Hochster and C. Huneke, Tight closure and strong F-regularity, Mem. Soc. Math. France 38 (1989), 119-133. MR 91i:13025

[HH1] - Tight closure, invariant theory, and the Briançon-Skoda theorem, J. Amer. Math. Soc. 3 (1990), 31-116. MR 91g:13010

[HH2] , F-regularity, test elements, and smooth base change, Trans. Amer. Math. Soc. 346 (1994), 1-62. MR 95d:13007

[HH3] , Tight closure in equal characteristic zero, to appear.

[How] J. A. Howald, Multiplier ideals of monomial ideals, Trans. Amer. Math. Soc. 353 (2001), 2665-2671. MR 2002b:14061

[Hu] C. Huneke, Tight closure and its applications, C.B.M.S. Regional Conference Series in Mathematics, No. 88, American Mathematical Society, Providence, RI, 1996. MR 96m:13001

[Hy1] E. Hyry, Blow-up rings and rational singularities, Manuscripta Math. 98 (1999), 377390. MR 2001d:13002

[Hy2] Coefficient ideals and the Cohen-Macaulay property of Rees algebras, Proc. Amer. Math. Soc. 129 (2001), 1299-1308. MR 2001h:13005

[Ka] Y. Kawamata, The cone of curves of algebraic varieties, Ann. Math. 119 (1984), 603633. MR 86c:14013b

$[\mathrm{Ku}] \quad$ E. Kunz, On Noetherian rings of characteristic p, Amer. J. Math. 98 (1976), 999-1013. MR 55:5612

[La] R. Lazarsfeld, Positivity in Algebraic Geometry, preprint.

[Li] Lipman, J., Adjoints of ideals in regular local rings, Math. Res. Letters 1 (1994), 739755. MR 95k:13028

[Mc] B. MacCrimmon, Weak F-regularity is strong F-regularity for rings with isolated non$\mathbb{Q}$-Gorenstein points, Trans. Amer. Math. Soc., to appear.

[Ma] H. Matsumura, Commutative ring theory, Cambridge Studies in Advanced Mathematics, vol. 8, Cambridge University Press, Cambridge, 1986. MR 88h:13001

[MS] V. B. Mehta and V. Srinivas, A characterization of rational singularities, Asian J. Math. 1 (1997), 249-278. MR 99e:13009

[N] A. Nadel, Multiplier ideal sheaves and Kähler-Einstein metrics of positive scalar curvature, Ann. Math. 132 (1990), 549-596. MR 92d:32038

[Si] A. K. Singh, F-regularity does not deform, Amer. J. Math. 121 (1999), 919-929. MR 2000e:13006

[Sm1] K. E. Smith, F-rational rings have rational singularities, Amer. J. Math. 119 (1997), 159-180. MR 97k:13004

[Sm2] _ The multiplier ideal is a universal test ideal, Comm. Algebra 28 (2000), 59155929. MR 2002d:13008 
[T] S. Takagi, An interpretation of multiplier ideals via tight closure, preprint.

[VV] P. Valabrega and G. Valla, Form rings and regular sequences, Nagoya Math. J. 72 (1978), 93-101. MR 80d:14010

[Vr] A. Vraciu, Strong test ideals, J. Pure Appl. Algebra 167 (2002), 361-373. MR 2003a:13004

[Wa] K.-i. Watanabe, F-regular and F-pure normal graded rings, J. Pure Appl. Algebra 71 (1991), 341-350. MR 92g:13003

[Wi] L. J. Williams, Uniform stability of kernels of Koszul cohomology indexed by the Frobenius endomorphism, J. Algebra 172 (1995), 721-743. MR 96f:13003

Mathematical Institute, Tohoku University, Sendai 980-8578, Japan

E-mail address: hara@math.tohoku.ac.jp

Graduate School of Mathematics, Nagoya University, Chikusa-Ku, Nagoya 464-8602, JAPAN

E-mail address: yoshida@math.nagoya-u.ac.jp 